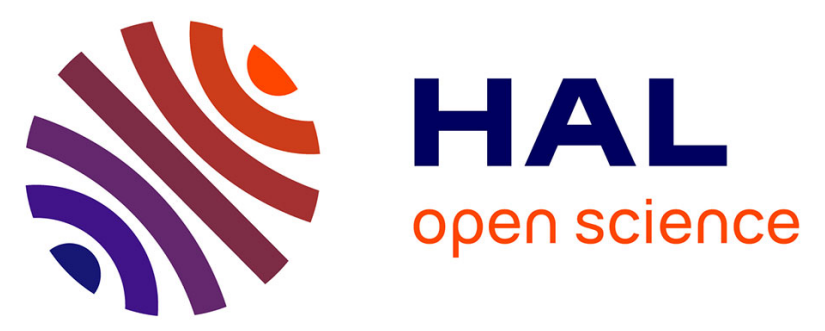

\title{
Toward an optimal a priori reduced basis strategy for frictional contact problems with LATIN solver
} Anthony Giacoma, David Dureisseix, Anthony Gravouil, Michel Rochette

\section{To cite this version:}

Anthony Giacoma, David Dureisseix, Anthony Gravouil, Michel Rochette. Toward an optimal a priori reduced basis strategy for frictional contact problems with LATIN solver. Computer Methods in Applied Mechanics and Engineering, 2015, 283, pp.1357-1381. 10.1016/j.cma.2014.09.005 . hal01090097

\section{HAL Id: hal-01090097 https://hal.science/hal-01090097}

Submitted on 15 Nov 2016

HAL is a multi-disciplinary open access archive for the deposit and dissemination of scientific research documents, whether they are published or not. The documents may come from teaching and research institutions in France or abroad, or from public or private research centers.
L'archive ouverte pluridisciplinaire HAL, est destinée au dépôt et à la diffusion de documents scientifiques de niveau recherche, publiés ou non, émanant des établissements d'enseignement et de recherche français ou étrangers, des laboratoires publics ou privés.

\section{(ㅇ)(1) $\$$}

Distributed under a Creative Commons Attribution - NonCommercial - NoDerivatives $\mid 4.0$ 


\title{
Toward an optimal a priori reduced basis strategy for frictional contact problems with LATIN solver
}

\author{
Anthony Giacoma ${ }^{1,2}$, David Dureisseix ${ }^{2}$, Anthony Gravouil ${ }^{2,3}$, and \\ Michel Rochette ${ }^{1}$ \\ ${ }^{1}$ ANSYS France, 11 Av. A. Einstein, F-69100 Villeurbanne, France \\ ${ }^{2}$ Univ Lyon, INSA Lyon, LaMCoS, CNRS UMR 5259, 18-20 Rue \\ des Sciences, F-69621 Villeurbanne Cedex, France \\ ${ }^{3}$ Institut universitaire de France
}

\begin{abstract}
In this paper, An efficient a priori model reduction strategy for frictional contact problems is presented. We propose to solve this problem by using the finite element method and the non-linear LATIN solver. Basically, this non-linear solver assumes a space time separated representation presaging nowadays PGD strategies. We extend this family of solvers to frictional engineering applications with reduced subspaces and no prior knowledge about the solution (contrary to a posteriori model reduction techniques). Hereinafter, a hybrid a priori/a posteriori LATINPGD formulation for frictional contact problems is proposed. Indeed, the suggested algorithm may or may not start with an initial guess of the reduced basis and is able to enrich the basis in order to reach a given level of accuracy. Moreover, it provides progressively the solution of the considered problem into a quasi-optimal space-time separated form compared to the singular value decomposition (SVD). Some examples are provided in order to illustrate the efficiency and quasi-optimality of the proposed a priori reduced basis LATIN solver.

This is a preprint of the following article: A. Giacoma, D. Dureisseix, A. Gravouil, M. Rochette, Toward an optimal a priori reduced basis strategy for frictional contact problems with LATIN solver, Computer Methods in Applied Mechanics and Engineering 283:1357-1381, 2015. DOI: 10.1016/j.cma.2014.09.005. (C) 2015, Elsevier. Licensed under the Creative Commons Attribution-NonCommercial-NoDerivatives 4.0 International http://creativecommons.org/licenses/by-nc-nd/4.0/
\end{abstract}

Classification. MSC: 74M10; 74M15; 65M60; 74S05

Keywords. Frictional contact; LATIN method; Reduced Order Model; Proper Generalized Decomposition (PGD) 


\section{Introduction}

Finite element simulations involving frictional contact dare the computational mechanics community. In spite of sophisticated algorithms, such simulations can lead to strong numerical difficulties and large computational cost. Contact mechanics is a broad topic involving several geometrical, optimization and numerical aspects relying on strong mathematical foundations. A great coverage of computational contact mechanics with all its basic ingredients is available in [80]: from contact detection to implementation of contact algorithms in a finite element software.

Frictional contact leads to a strong non-linear problem on the contacting interface. Indeed, such phenomenon is characterized by non-smooth constitutive laws (for both normal and tangential components). From various computational fields and over the last decade, numerous numerical methods were proposed to solve such problems. Generally speaking, contact problems can be formulated as an optimization problem subjected to constraints. Then, the Lagrange multiplier method can be used. For frictionless cases, such a formulation can be casted into a Linear Complementarity Problem and solved with dedicated approaches (Lemke's algorithm, projected successive over-relaxation, active-set methods...). For frictional cases, [9] proposes a method based on a discretization of the Coulomb's cone affecting toughly the precision of the solution. Augmented Lagrangian approaches are the most widespread formulations and many methods were proposed to solve both frictionless and frictional problems with these approaches $[42,76,60,79,48,2,22,69]$. Non-linear Gauss-Seidel solvers are also developed and applied to problems involving multi-contact bodies (rigid or flexible) $[53,51]$, but they are not usually the most efficient way to solve large systems due to a somehow low convergence rate. Gradient methods $[11,40,71,38]$ and generalized Newton's methods $[1,63,32]$ were also adapted to contact problems to reproduce performances of these well-known techniques. The non-smooth behavior of the frictional contact generally prevents to provide a convergence proof of these algorithms. Finally, the bipotential method [34, 52] provides a formulation and a suited algorithm allowing also some computer cost reduction. In [78], main results on space discretization scheme and non-linear solver for the solution of frictional contact problems are provided.

Usually and even within a quasi-static context, all these non-linear solvers may lead to a prohibitive computing time for industrial purposes. To address this computational issue, several acceleration methods can be used. First, an acceleration strategy based on multigrid methods were proposed in $[4,61]$. It consists in computing cheaply corrections on coarser discretizations (grids) to accelerate the convergence rate of a non-linear solver (e.g. non-smooth Newton method) also called smoother on the finest description of the problem. Second, domain decomposition techniques (FETI-based) were also proposed for frictional contact problems [36, 37]. Such techniques aims at partitioning bodies into nonoverlapping sub-domains processed independently. Then, interesting scalability property can be gained. On the other hand and over the last decade, model reduction techniques surge in enthusiasm within the computational mechanics field. For both linear and non-linear problems, impressive accelerations can be obtained. Nevertheless, for frictional contact mechanics, the use of such reduced basis methods seem to be difficult due the non-smoothness of the constitutive laws. As proposed and claimed in [45], using a space-time approach allows 


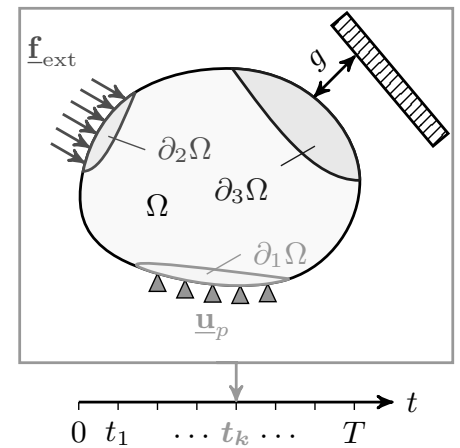

Figure 1: Problem set.

to circumvent this difficulty and benefiting from computational gain of such reduced basis approaches.

Classically, two different approaches are arising from model reduction techniques. Both consist in solving a given large scale reference problem in a reduced size subspace. The difference lies on the method providing the so-called Reduced Basis (RB). The first approach is called a posteriori method and consists firstly in designing a Reduced Order Model (ROM) [8, 6, 7, 23, 7, 66]. This is achieved by computing a RB with solution snapshots using e.g. Proper Orthogonal Decomposition (POD) or Singular Value Decomposition (SVD). Then, the reference problem and its governing equations are projected into the subspace engendered by a truncation of this RB. The ROM is built during an offline phase and may be expensive. The online phase consists in computing approximated solutions of the ROM. Such a technique aims to provide real-time and on-board computations and address efficiently multiparametric problems. Nevertheless, accuracy of the ROM depends strongly on the relevancy of the initial $\mathrm{RB}[50,49]$.

The second approach is the a priori method $[73,74]$. Contrary to the previous one, it does not require solution snapshots. The RB generating the approximation for the solution is computed along the resolution process. The best-known a priori method is the Proper Generalized Decomposition (PGD) $[28,29,26,59,16]$. It consists in searching the solution in a separable form reducing the computation cost. Moreover, the expensive offline phase is avoided.

In practice, a posteriori and a priori methods are complementary. The first approach approximates efficiently a given reference problem. The second one provides an efficient tool to adapt or enrich a given RB on-the-fly to generate suited approximations for the solution [54, 50, 43, 44].

In the present article, the authors propose an a priori approach close to the LATIN-PGD approach [57, 70,49] allowing to address frictional contact problems. This new non-linear solver uses ingredients from both the Large Time INcrement method (LATIN) [56] and the PGD. 


\section{Reference problem}

A flexible body is contacting a rigid basement (figure 1). We define $\Omega$, the domain occupied by this solid whose boundary is $\partial \Omega$ at time $t \in[0, T] \subset \mathbb{R}$. This boundary can be split into three complementary parts:

- $\partial_{1} \Omega$ : the part of the boundary where displacements $\mathbf{u}_{\mathrm{p}}$ are prescribed;

- $\partial_{2} \Omega$ : the part of the boundary where external loads $\mathbf{f}_{\text {ext }}$ are prescribed;

- $\partial_{3} \Omega$ : the part of the boundary where contact conditions may occur (potential contact interface).

\subsection{Strong formulation}

Linear assumptions are assessed (small displacements, homogeneous-isotropiclinear-elastic material, small sliding). External loads are time-dependent but a quasi-static regime is assumed. The problem can be set as follows: find the displacement field and the Cauchy's stress field denoted respectively by $\mathbf{u}(\mathbf{x}, t)$ and $\boldsymbol{\sigma}(\mathbf{x}, t)$ satisfying:

- kinematic admissibility:

$$
\mathbf{u}(\mathbf{x}, t) \in \mathcal{U}^{[0, T]} \text { and } \mathcal{U}^{[0, T]}=\left\{\mathbf{u} \in \mathcal{H}^{1}(\Omega) \text { with }\left.\mathbf{u}\right|_{\partial_{1} \Omega \times[0, T]}=\mathbf{u}_{\mathrm{p}}\right\}
$$

the strain-displacement (in small perturbations) relationship reads:

$$
\forall(\mathbf{x}, t) \in \Omega \times[0, T]: \quad \boldsymbol{\varepsilon}(\mathbf{u})=\frac{1}{2}\left(\boldsymbol{\nabla} \mathbf{u}+\boldsymbol{\nabla}^{T} \mathbf{u}\right)
$$

and the trace of the displacement field on the contact interface is $\mathbf{v}=$ $\left.\mathbf{u}\right|_{\partial_{3} \Omega \times[0, T]}$.

- static admissibility: $\boldsymbol{\sigma}(\mathbf{x}, t)$ is balanced with external force and contact forces $\lambda$, i.e.

$$
\left\{\begin{array}{lll}
\forall(\mathbf{x}, t) \in \partial_{2} \Omega \times[0, T]: & & \boldsymbol{\sigma} \mathbf{n}=\mathbf{f}_{\mathrm{ext}} \\
\forall(\mathbf{x}, t) \in \partial_{3} \Omega \times[0, T]: & & \boldsymbol{\sigma} \mathbf{n}=\lambda \\
\forall(\mathbf{x}, t) \in \Omega \times[0, T]: & & \operatorname{div}(\boldsymbol{\sigma})=\mathbf{0}
\end{array}\right.
$$

where $\mathbf{n}$ is the local outward normal vector.

- constitutive laws:

- Hooke law for elasticity:

$$
\forall(\mathbf{x}, t) \in \Omega \times[0, T]: \quad \boldsymbol{\sigma}=\mathbb{K} \varepsilon(\mathbf{u})
$$

where $\mathbb{K}$ is the linear elasticity operator.

- Coulomb frictional law for contact. With $\mathbf{v}$ and $\boldsymbol{\lambda}$, the traces of displacement field and contact force field (both normal and tangential) on the contacting interface $\partial_{3} \Omega$, these conditions can be written formally as in [35] as follows:

$$
\mathcal{R}(\mathbf{v}, \boldsymbol{\lambda})=0
$$


The fields at the contacting interface $\partial_{3} \Omega$ can be split into a normal and a tangential part as:

$$
\forall(\mathbf{x}, t) \in \partial_{3} \Omega \times[0, T]: \quad\left\{\begin{array}{l}
\mathbf{v}(\mathbf{x}, t)=u_{N} \mathbf{n}+\mathbf{u}_{T} \\
\boldsymbol{\lambda}(\mathbf{x}, t)=\lambda_{N} \mathbf{n}+\boldsymbol{\lambda}_{T}
\end{array}\right.
$$

According to Signorini conditions and Coulomb law on the displacement field and contact force field, operator $\mathcal{R}$ governs the contact behavior at the interface $\forall(\mathbf{x}, t) \in \partial_{3} \Omega \times[0, T]$

- Normal contact with Signorini conditions given in [75],

$$
\begin{aligned}
u_{N}=\mathbf{u} \cdot \mathbf{n} \leqslant g & \text { Non-penetration condition } \\
\lambda_{N}=(\boldsymbol{\sigma} \mathbf{n}) \cdot \mathbf{n} \leqslant 0 & \text { Compressive contact force } \\
\lambda_{N}\left(g-u_{N}\right)=0 & \text { Complementarity condition }
\end{aligned}
$$

where $g$ is the initial gap.

- Tangential contact with Coulomb friction law [31]:

$\left\|\boldsymbol{\lambda}_{T}\right\|_{2}=-\mu \lambda_{N} \quad$ if sliding: $\left\|\boldsymbol{\delta} \mathbf{u}_{T}\right\|_{2} \neq 0$ and $\boldsymbol{\delta} \mathbf{u}_{T}=-\rho \boldsymbol{\lambda}_{T}$ with $\rho>0$

$\left\|\boldsymbol{\lambda}_{T}\right\|_{2} \leqslant-\mu \lambda_{N} \quad$ if sticking: $\left\|\boldsymbol{\delta} \mathbf{u}_{T}\right\|_{2}=0$

where $\mu \in\left[0,+\infty\left[\right.\right.$ is the friction coefficient and $\delta \mathbf{u}_{T}$ is the increment of tangential displacement.

\subsection{Weak formulation}

We define the following set of homogeneous kinematically admissible functions as:

$$
\mathbf{u}(\mathbf{x}, t) \in \mathcal{U}_{0}^{[0, T]} \quad \text { with } \mathcal{U}_{0}^{[0, T]}=\left\{\mathbf{u} \in \mathcal{H}^{1}(\Omega) \text { and }\left.\mathbf{u}\right|_{\partial_{1} \Omega \times[0, T]}=\mathbf{0}\right\}
$$

Equilibrium (3) is equivalent to the following integral formulation with compatibility of contact force field and displacement field to contact conditions (7) and (8):

$$
\forall \mathbf{u}^{*} \in \mathcal{U}_{0}^{[0, T]}: \int_{\Omega} \boldsymbol{\sigma}: \boldsymbol{\varepsilon}\left(\mathbf{u}^{*}\right) \mathrm{d} V-\int_{\partial_{2} \Omega} \mathbf{f}_{\text {ext }} \cdot \mathbf{u}^{*} \mathrm{~d} S-\int_{\partial_{3} \Omega} \boldsymbol{\lambda} \cdot \mathbf{u}^{*} \mathrm{~d} S=0
$$

\subsection{Semi-discretized and discretized weak formulations}

Using Hooke law for $\boldsymbol{\sigma}=\mathbb{K} \varepsilon$ and the finite element approximation for displacement field yield to the so-called equilibrium equation with respect to frictional contact conditions at each contacting node:

$$
\begin{aligned}
& \forall \mathbf{u}^{*}(t): \int_{0}^{T} \mathbf{u}^{* T}(t) \mathbf{K u}(t) \mathrm{d} t \\
&=\int_{0}^{T} \mathbf{u}^{* T}(t)\left(\mathbf{f}_{\text {ext }}(t)+\mathbf{f}_{\mathrm{ctc}}(t)\right) \mathrm{d} t \text { with } \mathcal{R}=0
\end{aligned}
$$


$\mathbf{K}$ is the stiffness matrix, $\mathbf{f}_{\text {ext }}(t)$ and $\mathbf{f}_{\text {ctc }}(t)$ (corresponding to $\boldsymbol{\lambda}(t)$ ) are generalized forces and $\mathbf{B}$ is a boolean matrix mapping the global vector of nodal values to the values on contact boundary nodes. All in all, we have to find displacement field $\mathbf{u}(t)$ and contact force field $\mathbf{f}_{\mathrm{ctc}}(t)$ verifying (11) for all weighting functions $\mathbf{u}^{*}(t)$.

The time interval is discretized into a sequence of time steps $t_{1 \leqslant k \leqslant m}$ describing a regular time stepping (i.e. $t_{k+1}=t_{k}+\Delta t$ ). Then, the time-space discretized reference problem is defined as follows:

$$
\forall t \in\left[t_{1}, t_{m}\right]: \quad \mathbf{K u}=\mathbf{f}_{\text {ext }}+\mathbf{f}_{\text {ctc }} \quad \text { with } \quad \mathcal{R}=0
$$

This system characterized by stiffness matrix $\mathbf{K}$ has a size $n \times n$ (number of degrees of freedom) and has to be verified for $m$ time steps. The boolean mapping operator $\mathbf{B}$ is such that:

$$
\mathbf{v}=\mathbf{B u}=\left[\begin{array}{lll}
\mathbf{0} & \mathbf{1} & \mathbf{0} \\
\mathbf{0} & \mathbf{0} & \mathbf{1}
\end{array}\right]\left\{\begin{array}{c}
\overline{\mathbf{u}} \\
\mathbf{u}_{T} \\
\mathbf{u}_{N}
\end{array}\right\} \text { and } \mathbf{f}_{\mathrm{ctc}}=\mathbf{B}^{T} \boldsymbol{\lambda}=\left[\begin{array}{ll}
\mathbf{0} & \mathbf{0} \\
\mathbf{1} & \mathbf{0} \\
\mathbf{0} & \mathbf{1}
\end{array}\right]\left\{\begin{array}{c}
\boldsymbol{\lambda}_{T} \\
\boldsymbol{\lambda}_{N}
\end{array}\right\}
$$

Relationship 12 defines a general non-linear problem including a non-smooth constitutive law which can be extended to other behaviors without much change in the formulation (cohesive contact, etc.).

\section{Reduced basis and adaptation}

Reduced basis methods are attractive techniques to tackle computational cost and data storage issues. They are related to numerous techniques (principal component analysis, POD...) and are also precious tools for model reduction techniques.

\subsection{A posteriori approach and the LATIN/FAS principle}

Given a reference problem, a posteriori strategies consist in using prior knowledge about the solution to compute inexpensively other close solutions (e.g. multiparametric approach). This knowledge is obtained from some solution snapshots (or coarse meshes, analytic solutions...) and are compressed into a small basis using for instance SVD or POD [12]). Taking into account the most dominant directions, a RB is obtained. Then the equations of the problem can be Galerkin-projected into the RB providing the ROM for the considered problem. Efficiency of the ROM depends strongly on the relevancy of the RB. Thus, a compromise between precision and size of the ROM has to be reached.

In [45], analysis of RB provided by SVD are carried out for frictional contact problems and the LATIN/FAS strategy is proposed. It is exemplified that the $\mathrm{RB}$ has a multiscale content. First modes (the most energetic ones) are global at the overall structural scale, whereas higher order modes are localized to the contacting zone. The LATIN/FAS method proceeds as the non-linear FAS (Full Approximation Scheme) multigrid solver considering the RB as a coarse "grid". This strategy is embedded into the LATIN non-linear solver considered as a smoother. Coarse corrections are computed on the RB reducing error at the structural scale. Thus, the computational effort is concentrated to solve the 
localized frictional behavior. A consequent computational speedup is obtained and this strategy is efficient for multiparametric studies. Nevertheless, and in order to reach high levels of accuracy, the RB may not be able to produce relevant corrections. So, additional vectors are computed and added along the iterations to the RB (enrichment). Consequently, the main challenge of the LATIN/FAS is to provide a suited RB to compute relevant coarse corrections for iterated solution. Moreover, its suggested enrichment strategy may lead to expensive computations.

Hereinafter, the authors propose a robust PGD method consisting in solving the considered problem with an appropriate and evolving $\mathrm{RB}$ along iterations. Contrary to the LATIN/FAS method, the suggested a priori method does not require prior knowledge to design a suited $\mathrm{RB}$ which is computed on-the-fly and possibly from scratch. Furthermore, the strategy employed to achieve enrichment of the RB is far less expensive than the one proposed for the LATIN/FAS. In addition, it will be shown that the designed on-the-fly reduced basis has a quasi-optimal property. In other word, the computed RB is close to the best that one can compute for iterated solutions. Furthermore, as the PGD method is employed, a low rank approximation for the solution has to be handled. Consequently, a great computational work and huge memory requirements can be spared.

\subsection{A priori LATIN-PGD method}

Solution fields defined in (11) for all time steps can be collected into a $n$-by- $m$ snapshot matrix. For example, a snapshot matrix of the discretized displacement field is:

$$
\mathbf{U}=\left[\begin{array}{cccc}
u_{1}\left(t_{1}\right) & u_{1}\left(t_{2}\right) & \cdots & u_{1}\left(t_{m}\right) \\
u_{2}\left(t_{1}\right) & u_{2}\left(t_{2}\right) & \cdots & u_{2}\left(t_{m}\right) \\
\vdots & \vdots & \ddots & \vdots \\
u_{n}\left(t_{1}\right) & u_{n}\left(t_{2}\right) & \cdots & u_{n}\left(t_{m}\right)
\end{array}\right]=\left[\begin{array}{llll}
\mathbf{u}\left(t_{1}\right) & \mathbf{u}\left(t_{2}\right) & \cdots & \mathbf{u}\left(t_{m}\right)
\end{array}\right]
$$

The snapshot matrix (14) can be approximated using a separated form (or a low-rank approximation) as follows:

$$
\left\{\begin{array}{l}
\check{\mathbf{U}}=\mathbf{V}_{1} \boldsymbol{\Phi}_{1}^{T}+\mathbf{V}_{2} \boldsymbol{\Phi}_{2}^{T}+\cdots+\mathbf{V}_{p} \boldsymbol{\Phi}_{p}^{T} \\
\mathbf{u}(t)=\mathbf{V}_{1} \phi_{1}(t)+\mathbf{V}_{2} \phi_{2}(t)+\cdots+\mathbf{V}_{p} \phi_{p}(t)
\end{array}\right.
$$

The vectors $\mathbf{V}_{k}$ (respectively $\boldsymbol{\Phi}_{k}$ ) of size $n$ (respectively $m$ ) are associated to spatial modes (respectively temporal modes $\phi_{k}(t)$ ). The number of pairs of space-time modes (or dyads) is denoted by $p$. Consequently, a basis can be computed (e.g. a spatial basis) to approximate Ǔ. Such a basis can be considered as a RB for $\mathbf{U}$. In the following, we denote by $\left(\mathbf{V}_{k}\right)_{1}^{p}$ the $p$-tuple of space vectors and by $\left(\boldsymbol{\Phi}_{k}\right)_{1}^{p}$ the $p$-tuple of time vectors. If $p<1, p$-tuples is the empty set. The amount of data to store for (14) is of order $n \times m$ whereas for the low-rank form (15) it is $p(n+m)$. If $p<\frac{m n}{m+n}$, then low-rank form is advantageous. Generally speaking, $p$ has to be chosen such that the low-rank form (15) generates an accurate approximation for (14) keeping an attractive size for the expansion (15). 
The PGD method is introduced in [28]. It consists in building (15) with progressive enrichment stages (i.e. given an approximation $\check{\mathbf{U}}$, new vectors $\mathbf{V}$ and $\boldsymbol{\Phi}$ are sought and added to the expansion) making $\check{\mathbf{U}}$ more accurate and close to $\mathbf{U}$. This way and due to the fact that only vectors have to be computed and stored, computational work and memory usage is drastically reduced. Interested readers can also refer to [67] for exhaustive reviews and details.

\subsection{Optimality issues and downsizing}

Intrinsically, the PGD method does not ensure the optimality of the separated form (15) (i.e. it is a non-unique form and the optimal one is such that $p$ is minimal). According to [41] and considering the Frobenius norm, the best separated form is provided by the SVD (the so-called optimality property). To reach a certain level of accuracy for $\check{\mathbf{U}}$, PGD process may need numerous enrichment stages leading to large values for $p$ (with potentially memory overflow and computational cost issues). So for the sake of numerical efficiency, the size $p$ of (15) has to be controlled during the PGD process.

To enforce optimality property, a brute force solution is to perform a full SVD computation on $\check{\mathbf{U}}$ after each enrichment step [46, 10]. A less expansive method consists in deploying SVD updating techniques [21, 18]. Nevertheless, all of those lies on burdensome methods to compute precisely the SVD of the approximation $\check{\text { U. }}$ This high computational effort is not worthwhile knowing that $\check{\mathbf{U}}$ may be an inaccurate relatively to the solution of the reference problem.

This lead to assume that optimality property is useless and a soft downsizing approach suffices (i.e. maintain the quality of $\mathbf{U}$ while decreasing $p$ ). To design a suited algorithm, in a first step, an orthogonality property for $\left(\mathbf{V}_{k}\right)_{1}^{p}$ is considered (i.e. $\mathbf{V}_{i}^{T} \mathbf{V}_{j}=\boldsymbol{\delta}_{i j}$ with $\boldsymbol{\delta}_{i j}$ the Kronecker symbol). Then, the amplitude of each mode is attributed to the corresponding time vector $\left(\boldsymbol{\Phi}_{k}\right)_{1}^{p}$. To preserve this orthonormality property at each enrichment stage, one can use algorithm 1 .

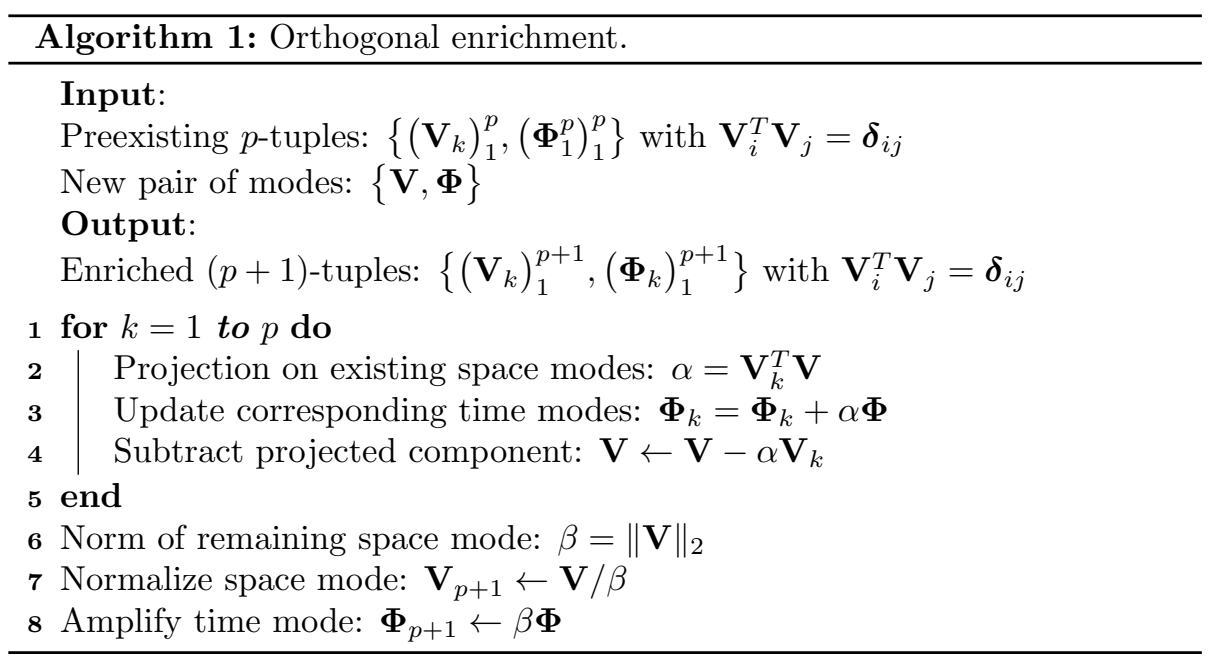

Notwithstanding orthonormality for $\left(\mathbf{V}_{k}\right)_{1}^{p}$, numerical experiments show that the inflation of the PGD basis is not stemmed, and a redundancy also 
occurs for the time modes $\left(\boldsymbol{\Phi}_{k}\right)_{1}^{p}$. To compress time vectors, the authors propose the following dyad to dyad projection strategy. In order to illustrate it, we consider an application for an approximation $\check{\mathbf{U}}$ written with two dyads:

$$
\check{\mathbf{U}}=\mathbf{V}_{1} \boldsymbol{\Phi}_{1}^{T}+\mathbf{V}_{2} \boldsymbol{\Phi}_{2}^{T} \quad \text { with } \quad \mathbf{V}_{i} \mathbf{V}_{j}=\boldsymbol{\delta}_{i j}
$$

Equation (16) can be rewritten considering that $\boldsymbol{\Phi}_{2}$ has a redundant component with $\boldsymbol{\Phi}_{1}$ (i.e. $\boldsymbol{\Phi}_{2}=\alpha \boldsymbol{\Phi}_{1}+\overline{\boldsymbol{\Phi}}_{2}$ and $\overline{\boldsymbol{\Phi}}_{2}^{T} \boldsymbol{\Phi}_{1}=0$ ) as follows:

$$
\check{\mathbf{U}}=\left(\mathbf{V}_{1}+\alpha \mathbf{V}_{2}\right) \boldsymbol{\Phi}_{1}^{T}+\mathbf{V}_{2} \overline{\boldsymbol{\Phi}}_{2}^{T}=\overline{\mathbf{V}}_{1} \boldsymbol{\Phi}_{1}^{T}+\mathbf{V}_{2} \overline{\mathbf{\Phi}}_{2}^{T}
$$

Rewriting (17) does not respect orthogonality for space vectors (i.e. $\mathbf{V}_{2}=$ $\beta \overline{\mathbf{V}}_{1}+\overline{\mathbf{V}}_{2}$ and $\left.\overline{\mathbf{V}}_{1}^{T} \overline{\mathbf{V}}_{2}=0\right)$. Applying a orthonormalization yields:

$$
\begin{aligned}
\check{\mathbf{U}}=\overline{\mathbf{V}}_{1}\left(\boldsymbol{\Phi}_{1}^{T}+\beta \overline{\mathbf{\Phi}}_{2}^{T}\right)+\overline{\mathbf{V}}_{2} \overline{\mathbf{\Phi}}_{2}^{T}= & \overline{\mathbf{V}}_{1} \overline{\mathbf{\Phi}}_{1}^{T}+\overline{\mathbf{V}}_{2} \overline{\mathbf{\Phi}}_{2}^{T} \\
& =\frac{\overline{\mathbf{V}}_{1}}{\left\|\overline{\mathbf{V}}_{1}\right\|}\left(\left\|\overline{\mathbf{V}}_{1}\right\| \overline{\mathbf{\Phi}}_{1}^{T}\right)+\frac{\overline{\mathbf{V}}_{2}}{\left\|\overline{\mathbf{V}}_{2}\right\|}\left(\left\|\overline{\mathbf{V}}_{2}\right\| \overline{\mathbf{\Phi}}_{2}^{T}\right)
\end{aligned}
$$

This process does not change $\check{\mathbf{U}}$ and preserves orthogonality property for space vectors but does not ensure it for time vectors. The first projection step (17) allows to compress redundancy between $\boldsymbol{\Phi}_{1}$ and $\boldsymbol{\Phi}_{2}$ into a new vector $\overline{\boldsymbol{\Phi}}_{1}$ but looses the orthogonality for space vectors, therefore justifying step (18). Incidentally, enforcing this property makes $\overline{\mathbf{\Phi}}_{1}$ and $\overline{\mathbf{\Phi}}_{2}$ no longer orthogonal. This process can be repeated until having $\overline{\boldsymbol{\Phi}}_{1}$ and $\overline{\boldsymbol{\Phi}}_{2}$ orthogonal (i.e. having produced the SVD of $\overline{\mathbf{U}}$ ).

Algorithm 2 generalizes this dyad to dyad process for larger expansions for U. The process $(17),(18)$ is applied in a certain sorting of modes, selected with time vectors amplitudes (i.e. the dyad with the weakest amplitude is projected on the one with the largest amplitude). 


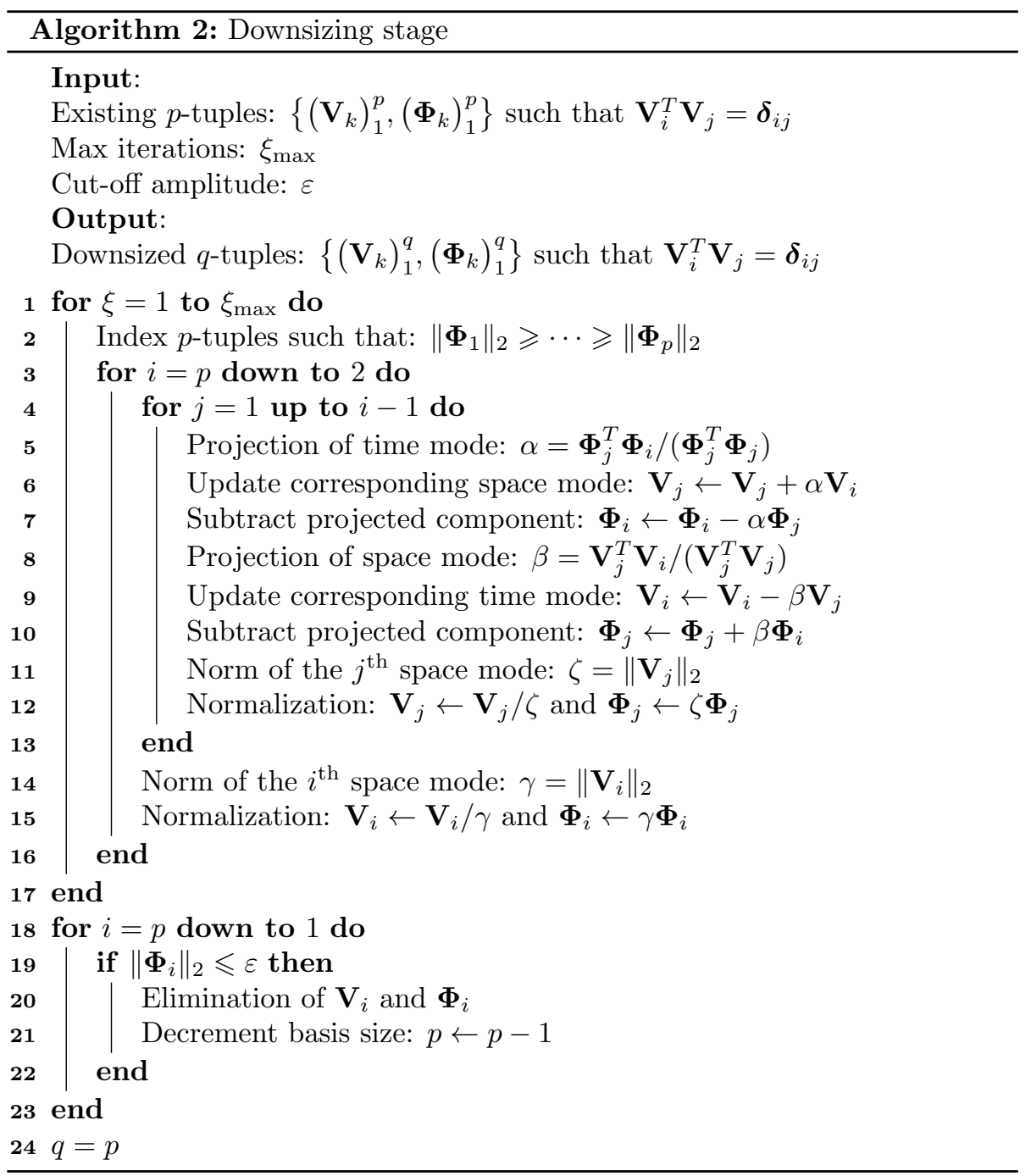

A complexity estimation, denoted by $c$ (floating operations), of algorithm 2 is $c / \xi_{\max }=n\left(\frac{5}{2} p^{2}-\frac{1}{2} p-2\right)+m\left(\frac{5}{2} p^{2}-\frac{3}{2} p-1\right)+\frac{3}{2} p^{2}-\frac{1}{2} p-1$. A cut-off amplitude $\varepsilon$ can be fixed relatively to a desired accuracy for $\mathbf{U}$. Embedded in the PGD process, the downsizing algorithm 2 stems efficiently the inflation of the expansion $\check{\mathbf{U}}$. In practice, a very few iterations $\xi_{\max }$ (1 or 2 iterations) is suf-

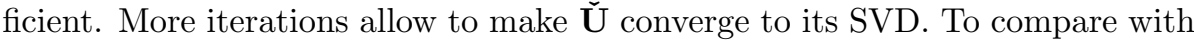
classical and widespread techniques: Lanczos thin-SVD method has a $\mathcal{O}\left(n m p^{2}\right)$ complexity [47] and fastest SVD updating methods have a complexity $\mathcal{O}(n m p)$ $[17]$.

\section{Formulation of the LATIN-PGD for frictional contact problems}

One of the major ingredients of the proposed strategy is the LATIN method [56]. This innovative non-incremental approach is well known for its ability to 
solve efficiently various non-linear time dependent problems such as frictional contact problems [24, 25, 45], large displacement [15], non-linear materials [13, 30, 70], transient dynamics [58, 62, 68]. The non-incremental LATIN method was initially proposed as a commitment of three principles:

(P1) Separation of the linear and non-linear behaviors. $\mathcal{A}$ denotes the set of solutions $\mathbf{s}=(\mathbf{v}, \boldsymbol{\lambda})$ satisfying linear constitutive law, kinematic admissibility and static admissibility. These are defined on the whole space-time domain $\Omega \times[0, T] . \quad \boldsymbol{\Gamma}$ denotes the set of solutions $\hat{\mathbf{s}}=(\hat{\mathbf{v}}, \hat{\boldsymbol{\lambda}})$ verifying frictional contact conditions and are defined locally at the contacting interface and on the whole time interval $\partial_{3} \Omega \times[0, T]$. The solution of the problem is $\mathbf{s} \in \mathcal{A} \cap \boldsymbol{\Gamma}$.

(P2) A two-staged iterative algorithm. The solution of the problem is searched with the construction of two sequences of approximations belonging alternatively to $\mathcal{A}$ and $\boldsymbol{\Gamma}$. At the $i^{\text {th }}$ iteration, the local stage consists in finding $\hat{\mathbf{s}}_{i}=\left(\hat{\mathbf{v}}_{i}, \hat{\boldsymbol{\lambda}}_{i}\right) \in \boldsymbol{\Gamma}$ with a search direction $\left(\hat{\mathbf{s}}_{i}-\mathbf{s}_{i-1}\right)=\left(\hat{\mathbf{v}}_{i}-\mathbf{v}_{i-1}, \hat{\boldsymbol{\lambda}}_{i}-\right.$ $\left.\boldsymbol{\lambda}_{i-1}\right) \in \mathbf{E}^{+}$. Note that $\mathbf{s}_{i-1}=\left(\hat{\mathbf{v}}_{i-1}, \hat{\boldsymbol{\lambda}}_{i-1}\right)$ is known from the previous iteration. Then, the global stage consists in finding $\mathbf{s}_{i}=\left(\mathbf{v}_{i}, \boldsymbol{\lambda}_{i}\right) \in \mathcal{A}$ with another search direction $\left(\mathbf{s}_{i}-\hat{\mathbf{s}}_{i}\right)=\left(\mathbf{v}_{i}-\hat{\mathbf{v}}_{i}, \boldsymbol{\lambda}_{i}-\hat{\boldsymbol{\lambda}}_{i}\right) \in \mathbf{E}^{-}$. Note that $\hat{\mathbf{s}}_{i}=\left(\hat{\mathbf{v}}_{i}, \hat{\boldsymbol{\lambda}}_{i}\right)$ is known from the previous local stage.

(P3) Radial approximation or space-time separation. Unknowns are represented as a sum of products between a space function and a time function. This supplementary constraint makes the problem over-determined. In order to enforce the constraints in $(\mathrm{P} 1)$, the search direction equation $\mathbf{E}^{-}$ is then verified in a weak sense. It was initially stated to limit memory usage.

These principles are illustrated on figure 2. For certain cases and for sake of simplicity, the LATIN method can be formulated without the space-time separation stated in (P3). In this case several similarities can be stated with augmented Lagrangian methods [3].

Local stage Given a solution $\mathbf{s}=(\mathbf{v}, \boldsymbol{\lambda})$ verifying the internal balance, kinematic admissibility and static admissibility, the local stage is an updating stage occurring at the contacting interface. At each node, non-linear frictional contact conditions and search direction equation have to be verified. The nodal search direction equations for both normal and tangential components reads:

$$
\left(\mathbf{E}^{+}\right):\left\{\begin{array}{l}
\hat{c}_{N}=\hat{\lambda}_{N}-\lambda_{N}=k_{N}\left(\hat{v}_{N}-v_{N}\right) \\
\hat{\mathbf{c}}_{T}=\hat{\boldsymbol{\lambda}}_{T}-\boldsymbol{\lambda}_{T}=k_{T}\left(\hat{\mathbf{v}}_{T}-\mathbf{v}_{T}\right)
\end{array}\right.
$$

$k_{N}$ and $k_{T}$ are parameters of the method defined at each node. It is similar to the penalty parameter in an augmented Lagrangian formulation. Different values for the normal and tangential problem can be chosen. These values influence only the convergence rate and optimal values are related to mechanical properties of the studied body [56, 25, 14, 77]. According to [14], close-to-optimum values are searched as follows:

$$
k_{N}=E L_{c} \quad \text { and } \quad k_{T}=E L_{c}^{\prime}
$$




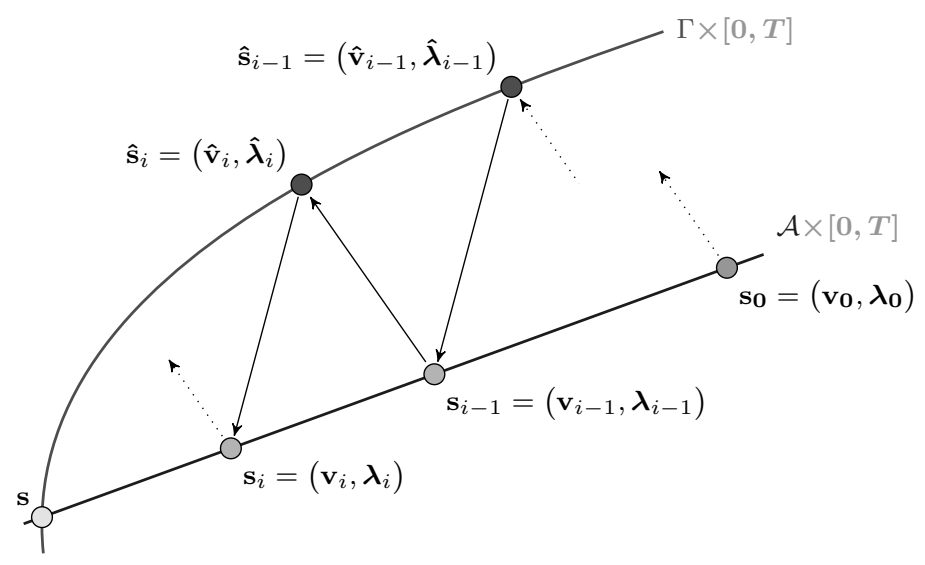

(a)

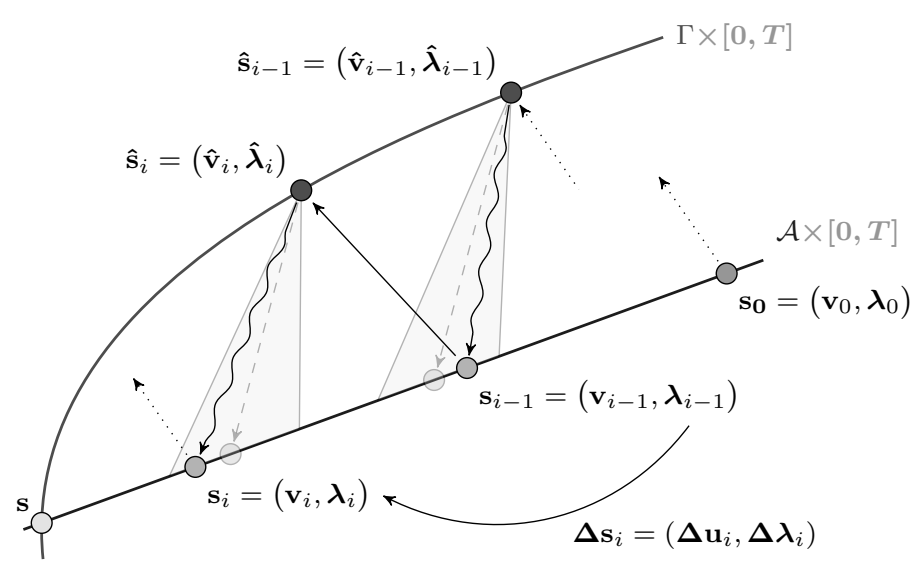

(b)

Figure 2: Illustration of LATIN non-linear iterative solver. (a) using (P1) and (P2) principles - (b) using (P1), (P2) and (P3) principles: dashed arrows symbolize exact search directions whereas waving line are approximated search directions. 


\begin{tabular}{|c|c|}
\hline \multicolumn{2}{|c|}{ Normal components } \\
\hline$\hat{\lambda}_{N}=\left\langle c_{N}\right\rangle$ & $\hat{v}_{N}=g-\frac{1}{k_{N}}\left\langle c_{N}\right\rangle_{+}$ \\
\hline \multicolumn{2}{|c|}{ Tangential components } \\
\hline Sliding: $\left\|\mathbf{c}_{T}\right\|_{2}>\mu\left|\hat{\lambda}_{N}\right|$ & Sticking: $\left\|\mathbf{c}_{T}\right\|_{2} \leqslant \mu\left|\hat{\lambda}_{N}\right|$ \\
\hline $\begin{array}{c}\mathbf{t}=\mathbf{c}_{T} /\left\|\mathbf{c}_{T}\right\|_{2} \\
\hat{\boldsymbol{\lambda}}_{T}=\mu\left|\hat{\lambda}_{N}\right| \mathbf{t} \\
\hat{\mathbf{v}}_{T}^{k}=\hat{\mathbf{v}}_{T}^{k-1}-\frac{1}{k_{T}}\left(\left\|\mathbf{c}_{T}\right\|_{2}-\mu\left|\hat{\lambda}_{N}\right|\right) \mathbf{t}\end{array}$ & $\begin{array}{c}\hat{\boldsymbol{\lambda}}_{T}=\mathbf{c}_{T} \\
\hat{\mathbf{v}}_{T}^{k}=\hat{\mathbf{v}}_{T}^{k-1}\end{array}$ \\
\hline
\end{tabular}

Table 1: Solutions of the local stage.

with $E$ the Young's modulus and $L_{c}$ contact characteristic dimensions. $k_{N, T}$ have the dimension of a stiffness. Search direction equations can be equivalently rewritten introducing the gap parameter (for the normal component) and the displacement of the previous time step (for the tangential component, with a superscript $k-1)$. This yields to the following equations:

$$
\left\{\begin{array}{l}
c_{N}=\hat{c}_{N}+k_{N} g=\lambda_{N}-k_{N}\left(v_{N}-g\right)=\hat{\lambda}_{N}-k_{N}\left(\hat{v}_{N}-g\right) \\
\mathbf{c}_{T}=\hat{\mathbf{c}}_{T}+k_{T} \hat{\mathbf{v}}_{T}^{k-1}=\boldsymbol{\lambda}_{T}-k_{T}\left(\mathbf{v}_{T}^{k}-\hat{\mathbf{v}}_{T}^{k-1}\right)=\hat{\boldsymbol{\lambda}}_{T}-k_{T}\left(\hat{\mathbf{v}}_{T}^{k}-\hat{\mathbf{v}}_{T}^{k-1}\right)
\end{array}\right.
$$

$\mathbf{v}_{T}^{k}$ refers to the nodal displacement on the contacting interface in the tangential plane at time step $t_{k}$. Then, contact conditions can be easily applied on each term of $c_{N}$ and $\mathbf{c}_{T}$.

Explicit solutions of the local stage are given in table 1 and have to be computed for each node belonging to the contacting interface and for each time step. Hence, the solution of the local stage $\hat{\mathbf{s}}=(\hat{\mathbf{v}}, \hat{\boldsymbol{\lambda}})$ verifies strictly frictional contact conditions and gives a prediction/correction given a solution $\mathbf{s}=(\mathbf{v}, \boldsymbol{\lambda})$ verifying internal balance. Moreover, $\hat{\mathbf{s}}$ is computed explicitly.

Global stage Given a solution $\hat{\mathbf{s}}=(\hat{\mathbf{v}}, \hat{\boldsymbol{\lambda}})$ issued from the previous local stage and verifying frictional contact conditions. The global stage consists in finding a space-time solution defined on the whole space domain and time interval verifying the linear constitutive law, kinematic admissibility, static admissibility. We introduce an additional search direction equation to introduce the local stage frictional contact prediction. This stage consists in finding a displacement field and a contact force field $\mathbf{s}=(\mathbf{v}, \boldsymbol{\lambda})$ defined over $\partial_{3} \Omega \times[0, T]$. These fields have to verify the admissibility equations:

$$
\left\{\begin{array}{l}
\mathbf{K u}=\mathbf{f}_{\text {ext }}+\mathbf{B}^{T} \boldsymbol{\lambda} \\
\mathbf{v}=\mathbf{B u}
\end{array}\right.
$$

$\mathbf{v}$ is the trace of the displacement field over $\partial_{3} \Omega \times[0, T]$ whereas $\mathbf{u}$ is defined over the whole space-time domain. According to the second principle of the 
LATIN method, the search direction has to be verified as well. Let us write search direction equations for the whole contacting interface (with $\mathbf{I}_{\mathbf{d}}$ the identity matrix):

$$
\left(\mathbf{E}^{-}\right):\left\{\begin{array}{l}
\boldsymbol{\lambda}_{N} \\
\boldsymbol{\lambda}_{T}
\end{array}\right\}-\left\{\begin{array}{l}
\hat{\boldsymbol{\lambda}}_{N} \\
\hat{\boldsymbol{\lambda}}_{T}
\end{array}\right\}=\underbrace{\left[\begin{array}{cc}
k_{N} \mathbf{I}_{\mathbf{d}} & \mathbf{0} \\
\mathbf{0} & k_{T} \mathbf{I}_{\mathbf{d}}
\end{array}\right]}_{\mathbf{k}}\left(\left\{\begin{array}{c}
\hat{\mathbf{v}}_{N} \\
\hat{\mathbf{v}}_{T}
\end{array}\right\}-\left\{\begin{array}{l}
\mathbf{v}_{N} \\
\mathbf{v}_{T}
\end{array}\right\}\right)
$$

All in all, taking into account only the first and the second principle of the LATIN method leads to solve the following linear system for each time step:

$$
\left\{\begin{array}{l}
{\left[\mathbf{K}+\mathbf{B}^{T} \mathbf{k B}\right] \mathbf{u}=\mathbf{f}_{\text {ext }}+\mathbf{B}^{T}(\hat{\boldsymbol{\lambda}}+\mathbf{k} \hat{\mathbf{v}})} \\
\mathbf{v}=\mathbf{B u} \\
\boldsymbol{\lambda}=\hat{\boldsymbol{\lambda}}+\mathbf{k}(\hat{\mathbf{v}}-\mathbf{v})
\end{array}\right.
$$

Note that the operator $\left[\mathbf{K}+\mathbf{B}^{T} \mathbf{k B}\right]$ is symmetric definite positive and remains constant along the iterations.

$\mathbf{K}$ is the stiffness matrix, taking into account the Dirichlet boundary conditions (1). If these last conditions are not sufficient, $\mathbf{K}$ is only semi-definite positive. If the genuine problem is well-posed, the active and/or sticking contact conditions for the solution are sufficient to prevent any undefined rigid body motion. The full matrix $\left[\mathbf{K}+\mathbf{B}^{T} \mathbf{k B}\right]$ is the previous stiffness matrix completed by a Robin-like boundary condition (with stiffness $\mathbf{k}$ ) on the whole potential contact area. This last term is therefore sufficient to get a regular left hand side. Nevertheless, one has to take care of its condition number to solve efficiently such a linear system.

Initialization and stopping criterion Assuming that $\mathbf{s} \in \mathcal{A}$, so does the initial guess $\mathbf{s}_{0}$. Consequently, the iterative process is initialized with the linear contactless elastic solution:

$$
\mathbf{s}_{0}=\left(\mathbf{u}_{0}, \boldsymbol{\lambda}_{0}\right) \quad \text { such that }\left\{\begin{array}{l}
\mathbf{K} \mathbf{u}_{0}=\mathbf{f}_{\text {ext }} \\
\boldsymbol{\lambda}_{0}=\mathbf{0}
\end{array}\right.
$$

We also use the convergence indicator (26) introduced in [72]. Iterations are stopped once a threshold is reached: $\mathcal{I}<\varepsilon$.

$$
\begin{array}{r}
\mathcal{I}=\max \left(\sqrt{\frac{\left\|\mathbf{s}_{N}-\hat{\mathbf{s}}_{N}\right\|_{\infty}^{2}}{\left\|\mathbf{s}_{N}\right\|_{\infty}^{2}+\left\|\hat{\mathbf{s}}_{N}\right\|_{\infty}^{2}}}, \sqrt{\frac{\left\|\mathbf{s}_{T}-\hat{\mathbf{s}}_{T}\right\|_{\infty}^{2}}{\left\|\mathbf{s}_{T}\right\|_{\infty}^{2}+\left\|\hat{\mathbf{s}}_{T}\right\|_{\infty}^{2}}}\right) \\
\text { with }\left\|\mathbf{s}_{N, T}\right\|_{\infty}^{2}=\max _{\mathbf{x}, t}\left[\frac{1}{k_{N, T}} \lambda_{N, T}^{2}+k_{N, T} u_{N, T}^{2}\right]
\end{array}
$$

where $k_{N, T}$ is the search direction parameter. Note that subscripts $N$ and $T$ refer respectively to normal and tangential components at a given node belonging to the contacting interface. Using maximum norms, the convergence criterion (26) is a very tough criterion. A comparison with an other far less restrictive indicator is available in [45]. 
Global stage with space-time separation The third principle consists in searching the solution of the reference problem (both displacement field and contact force field) under a separated form. Using the finite element framework, it reads:

$$
\begin{aligned}
& \mathbf{u}(t)=\mathbf{u}_{0}(t)+\sum_{k=1}^{p} \mathbf{V}_{k} \phi_{k}(t) \\
& \boldsymbol{\lambda}(t)=\boldsymbol{\lambda}_{0}(t)+\sum_{k=1}^{p} \mathbf{L}_{k} \psi_{k}(t)
\end{aligned}
$$

Vectors $\mathbf{V}_{k}$ (resp. $\mathbf{L}_{k}$ ) are space vectors associated to the displacement field defined over $\Omega$ (resp. contact force field defined over $\partial_{3} \Omega$ ) and functions $\phi_{k}(t)$ (resp. $\left.\psi_{k}(t)\right)$ are time functions associated to $\mathbf{V}_{k}$ (resp. $\mathbf{L}_{k}$ ) and defined on $[0, T]$. Vectors $\mathbf{u}_{0}$ and $\boldsymbol{\lambda}_{0}$ are initial guesses (which can be also provided as low rank approximations). The approximation (27) can be written equivalently using a matrix notation as follows:

$$
\begin{gathered}
\mathbf{u}=\mathbf{u}_{0}+\sum_{k=1}^{p} \mathbf{V}_{k} \boldsymbol{\Phi}_{k}^{T} \\
\boldsymbol{\lambda}=\boldsymbol{\lambda}_{0}+\sum_{k=1}^{p} \mathbf{L}_{k} \boldsymbol{\Psi}_{k}^{T}
\end{gathered}
$$

First, we reformulate equations of the global stage with a corrective scheme. At a given iteration (indexed by $i)$, the correction $\Delta \mathbf{s}=(\boldsymbol{\Delta} \mathbf{u}, \boldsymbol{\Delta} \boldsymbol{\lambda})$ is computed and added to the solution of the previous global stage $\mathbf{s}_{i-1}$, so that the quality of the updated solution $\mathbf{s}=\mathbf{s}_{i-1}+\Delta \mathbf{s}$ improves. Subtracting appropriate quantities to equations (24) yields to the following problem to solve:

$$
\left\{\begin{array}{l}
\mathbf{K} \boldsymbol{\Delta} \mathbf{u}=\mathbf{B}^{T} \boldsymbol{\Delta} \lambda \\
\Delta \mathbf{v}=\mathbf{B} \boldsymbol{\Delta} \mathbf{u} \\
\Delta \boldsymbol{\lambda}+\mathbf{k} \boldsymbol{\Delta} \mathbf{v}-\operatorname{res}_{\mathrm{sd}}=0
\end{array}\right.
$$

where $\operatorname{res}_{\mathrm{sd}}=\hat{\boldsymbol{\lambda}}-\boldsymbol{\lambda}_{i-1}+\mathbf{k}\left(\hat{\mathbf{v}}-\mathbf{v}_{i-1}\right)$ is known at this stage.

Second, we introduce the separated representation for corrective increments:

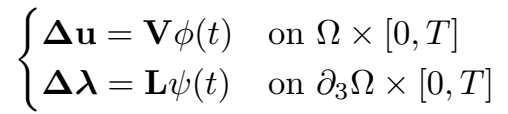

Substituting representations (30) in the internal balance equation of (29) yields to a relationship between space and time functions:

$$
\forall t \in[0, T]: \mathbf{K V} \phi(t)=\mathbf{B}^{T} \mathbf{L} \psi(t) \Leftrightarrow\left\{\begin{array}{l}
\mathbf{K V}=\mathbf{B}^{T} \mathbf{L} \\
\phi(t)=\psi(t)
\end{array}\right.
$$

The problem (29) including representation (30) respecting (31) (i.e. internal balance equation) is over-constrained. As a consequence, search direction equations are verified at best (i.e. in a weak sense) by solving the following general minimization problem:

$$
\{\mathbf{W}, \phi(t)\}=\arg \min _{\overline{\mathbf{W}}, \bar{\phi}(t)}\left\|\overline{\mathbf{W}} \bar{\phi}(t)-\mathbf{r e s}_{\mathrm{sd}}\right\|_{F}
$$


with $\mathbf{W}=\mathbf{L}+\mathbf{k B V}$. The auxiliary space unknown $\mathbf{W}$ defined on $\partial_{3} \Omega$ is introduced to take into account the linear relationship (31) between $\mathbf{V}$ and $\mathbf{L}$ ensuring the internal balance. To compute them, the following relationships can be used:

$$
\left\{\begin{array}{l}
{\left[\mathbf{K}+\mathbf{B}^{T} \mathbf{k B}\right] \mathbf{V}=\mathbf{B}^{T} \mathbf{W}} \\
\mathbf{L}=\mathbf{W}-\mathbf{k B V}
\end{array}\right.
$$

\subsection{Find time functions with fixed space functions}

In this stage, one looks for solving global stage defined in (32) with given space functions $\left(\mathbf{W}_{k}\right)_{1}^{p}$ provided by an initial guess or by the previous iterations. Corrections $\left(\widetilde{\phi}_{k}\right)_{1}^{p}$ for time functions are sought according the following optimization problem:

$$
\widetilde{\phi}_{k}=\underset{\bar{\phi}}{\operatorname{argmin}}\left\|\mathbf{W}_{k} \bar{\phi}-\operatorname{res}_{\mathrm{sd}}\right\|_{F}
$$

A Galerkin method is applied with the trial function $\boldsymbol{\omega}^{\star}=\mathbf{W}_{k} \tilde{\phi}^{\star}(t)$ and yields to:

$$
\widetilde{\phi}_{k}=\mathbf{W}_{k}^{T} \mathbf{r e s}_{\mathrm{sd}}
$$

Consequently, updated time modes are $\left(\phi_{k}\right)_{1}^{p} \leftarrow\left(\phi_{k}+\widetilde{\phi}_{k}\right)_{1}^{p}$. The corresponding pseudo-code is given in algorithm 3 .

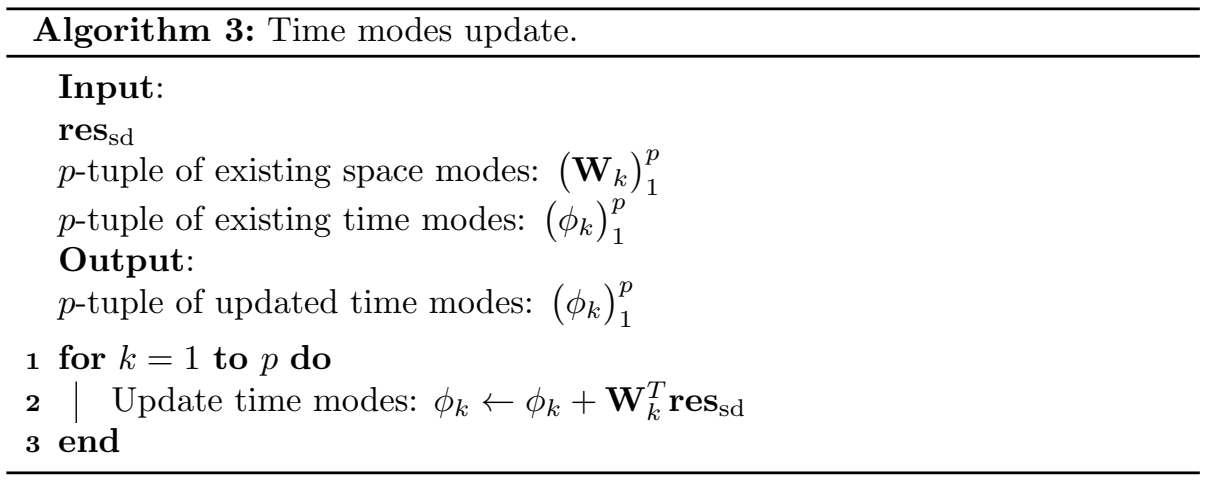

Because space functions are fixed, convergence may be slow and accuracy for the solution depends the given space basis. Usually, this stage is only deployed to update time functions and does not suffice to solve the considered problem. To make the space basis more relevant, extra space modes have to be computed and added.

\subsection{Enrichment stage}

The enrichment stage consists in generating a new pair of space-time functions extending the basis and making the iterated solution more accurate. So, the following optimization problem for the global stage defined on (32) has to be solved:

$$
\{\mathbf{W}, \phi(t)\}=\underset{\overline{\mathbf{W}} \bar{\phi}(t)}{\operatorname{argmin}}\left\|\overline{\mathbf{W}} \bar{\phi}(t)-\mathbf{r e s}_{\mathrm{sd}}\right\|_{F}
$$


Based on a space-time weak form modeling method as (11), a Galerkin method is applied with the trial function $\boldsymbol{\omega}^{\star}=\mathbf{W}^{\star} \phi(t)+\mathbf{W} \phi^{\star}(t)$ and yields to:

$$
\int_{0}^{T} \boldsymbol{\omega}^{\star}\left(\mathbf{W} \phi(t)-\mathbf{r e s}_{\mathrm{sd}}\right) \mathrm{d} t=0
$$

The non-linear system (37) is solved with a robust iterative strategy [27]. It proceeds in two steps, repeated until convergence. The first step consists in computing vector $\mathbf{W}$ knowing $\phi(t)$ from the previous step. Then, the second step consists in updating $\phi(t)$ knowing $\mathbf{W}$ from the first step. The process is stopped when $\mathbf{W}$ and $\phi(t)$ are no more significantly updated.

First step Space vector $\mathbf{W}$ is updated knowing $\phi(t)$ from the previous step:

$$
\mathbf{W}=\int_{0}^{T} \operatorname{res}_{\mathrm{sd}} \phi(t) \mathrm{d} t / \int_{0}^{T} \phi^{2}(t) \mathrm{d} t
$$

Second step Time function $\phi(t)$ is searched knowing $\mathbf{W}$ from the first step:

$$
\phi(t)=\mathbf{W}^{T} \mathbf{r e s}_{\mathrm{sd}} /\left(\mathbf{W}^{T} \mathbf{W}\right)
$$

In practice, a very few iterations are needed and a normalization condition is applied for one of the two vectors. The pseudo-code of this method is given in the algorithm 4.

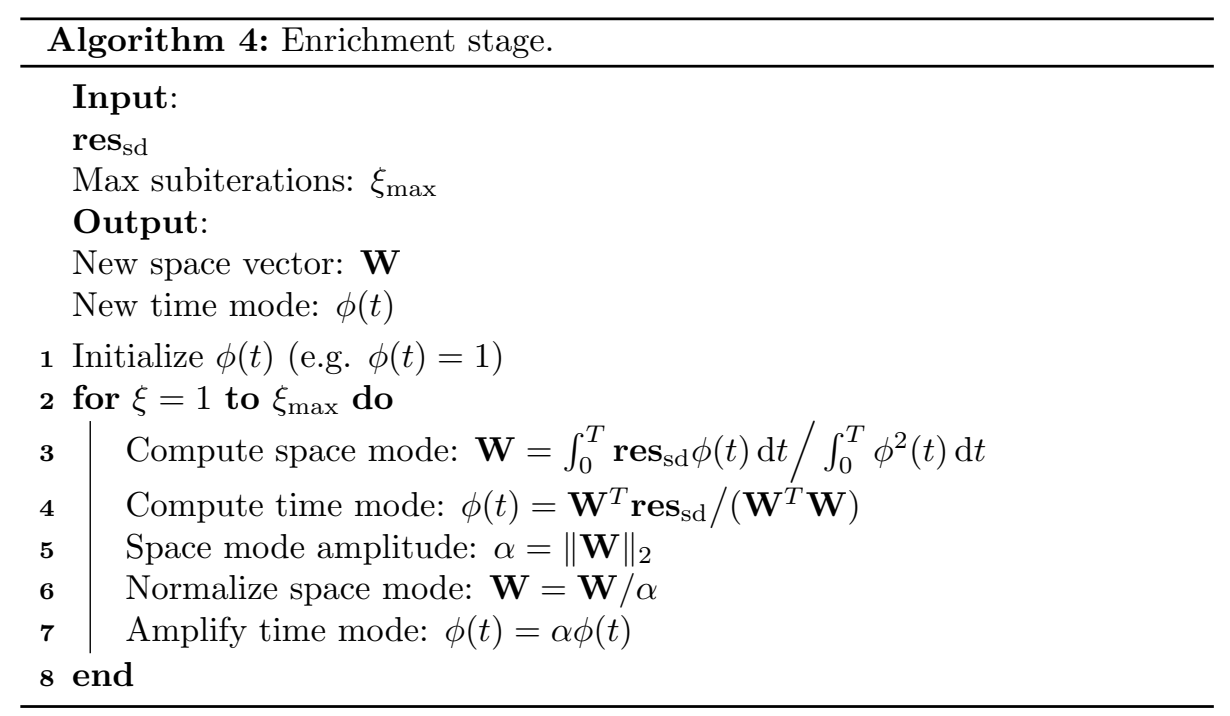

Once the new pair is found, space and time basis are enriched using algorithm 1.

\subsection{Preliminary stage}

In [13], the preliminary stage consists in updating time functions as described in 4.1. Then, the enrichment stage is used only if the convergence criterion stagnates (e.g. if the error indicator decreases by a factor less than 10\%). We 
propose herein to enhance the preliminary stage by updating space modes as well with corrections $\left(\widetilde{\mathbf{W}}_{k}\right)_{1}^{p}$ for fixed time modes. This new updating phase is defined from the global stage equation (32) and the following optimization problem:

$$
\widetilde{\mathbf{W}}_{k}=\underset{\overline{\mathbf{W}}}{\operatorname{argmin}}\left\|\overline{\mathbf{W}} \phi_{k}(t)-\mathbf{r e s}_{\mathrm{sd}}\right\|_{F}
$$

A Galerkin method with the trial function $\boldsymbol{\omega}^{\star}=\mathbf{W}_{k}^{\star} \phi_{k}(t)$ is used and yields to:

$$
\widetilde{\mathbf{W}}_{k}=\int_{0}^{T} \operatorname{res}_{\mathrm{sd}} \phi_{k}(t) \mathrm{d} t / \int_{0}^{T} \phi_{k}^{2}(t) \mathrm{d} t
$$

Because of orthonormality condition, space modes $\left(\mathbf{W}_{k}\right)_{1}^{p}$ can not be updated directly and each correction $\widetilde{\mathbf{W}}_{k}$ has to be projected in the spatial basis. The corresponding pseudo-code is given in algorithm 5 .

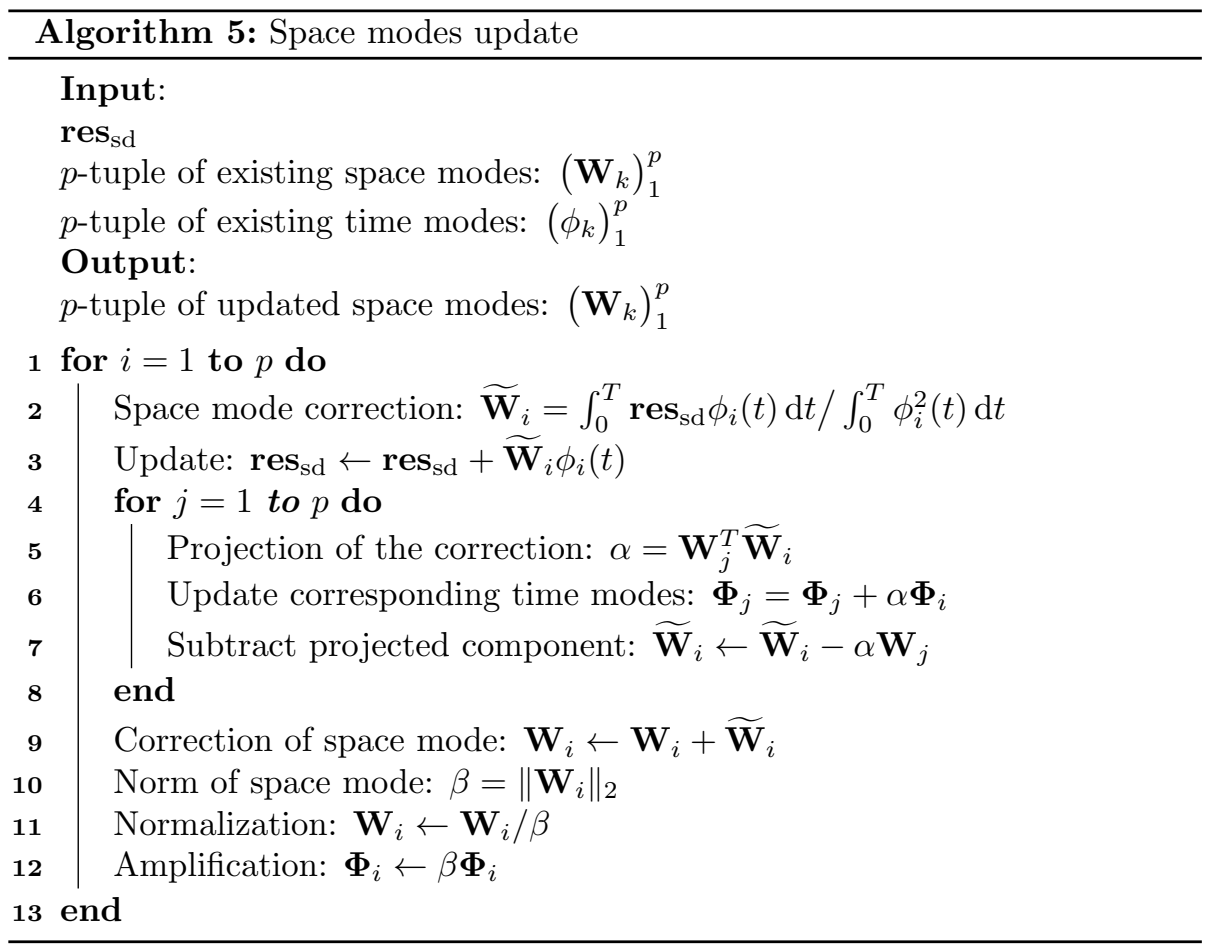

After the two updating stages for time and space modes, the enrichment stage is eventually used according to the decreasing of the convergence indicator. These stages fulfill the global stage and provide a kinematically and statically admissible solution. In addition, and once a global stage is solved, a downsizing stage defined with algorithm 2 is used in order to control the basis size.

\subsection{The quasi-optimal LATIN-PGD algorithm for frictional contact}

The whole pseudo-code of the quasi-optimal LATIN-PGD is given in algorithm 6. Note that, the cut-off amplitude criterion of the downsizing stage is choosen in relation to the LATIN-PGD convergence indicator. 


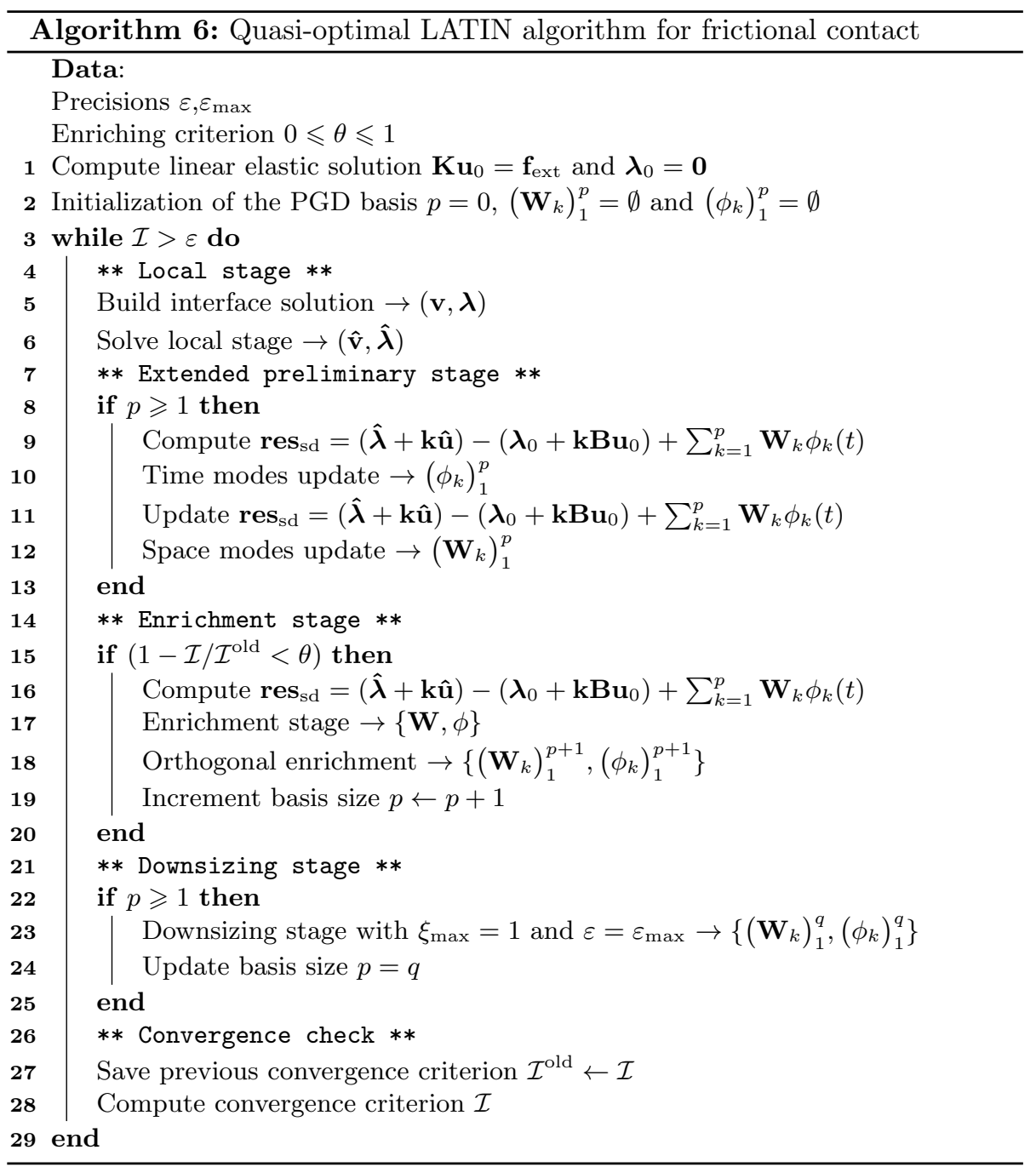

\section{Two-dimensional numerical application}

The proposed LATIN-PGD method is applied to the two dimensional frictional model illustrated in figure 3 with numerical properties given in Table 2 . It is a flexible indenter subject to prescribed displacements. These are designed such that boundary $\partial_{3} \Omega$ is contacting a rigid part with successively different regimes (active contact or not, sliding, sticking) within the studied time interval.

To solve the initial reference problem, the LATIN non-linear solver is used.

\subsection{A posteriori analysis of the reference solution}

As in [45], a posteriori analyses can be carried out to exemplify the reducibility of the space-time reference solution $\left(\mathbf{u}^{\mathrm{r}}, \boldsymbol{\lambda}^{\mathrm{r}}\right)$ and its multiscale content. Moreover, a SVD basis of reference vectors $\left(\mathbf{W}_{k}^{\mathrm{r}}\right)_{1}^{P}$ can be extracted from the following singular values decomposition: 


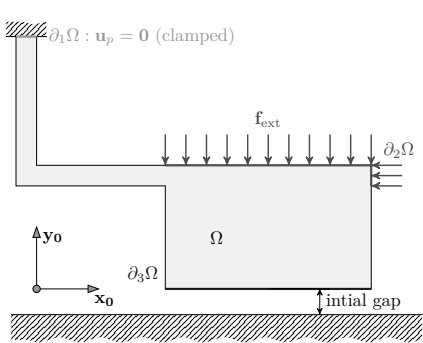

(a)

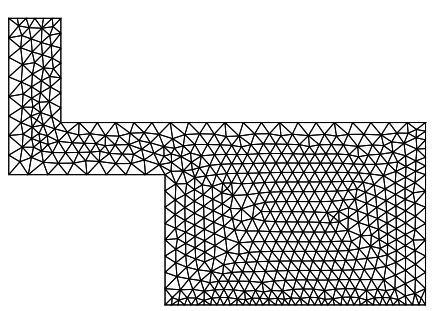

(c)

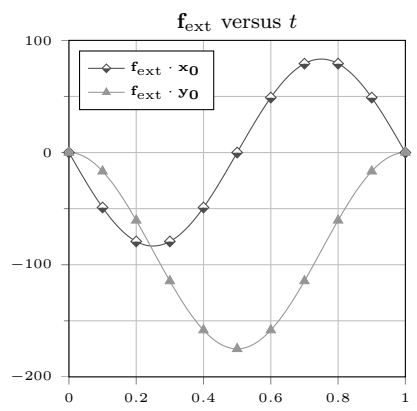

(b)

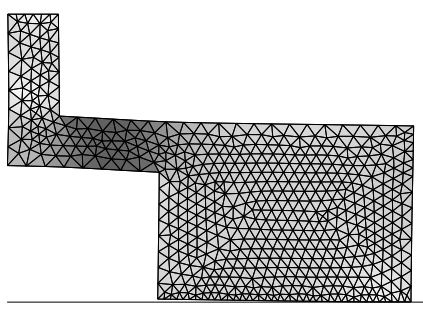

(d)

Figure 3: (a) Model — (b) Prescribed displacement evolution - (c) Mesh (d) Shape of the deformed body at $t_{150}$.

\begin{tabular}{|c|c|c|}
\hline Number of time steps & $m$ & 200 \\
\hline Number of dofs & $n$ & 1194 \\
\hline Number of contacting nodes & $n_{C}$ & 41 \\
\hline Young modulus & $E$ & $10^{6} \mathrm{~Pa}$ \\
\hline Poisson ratio & $\nu$ & 0.3 \\
\hline Friction coefficient & $\mu$ & 0.15 \\
\hline Final time & $T$ & $1 \mathrm{~s}$ \\
\hline Search direction & $k_{T}$ & $40000 \mathrm{~N} / \mathrm{m}$ \\
\hline Search direction & $k_{N}$ & $20000 \mathrm{~N} / \mathrm{m}$ \\
\hline
\end{tabular}

Table 2: Simulation parameters. 


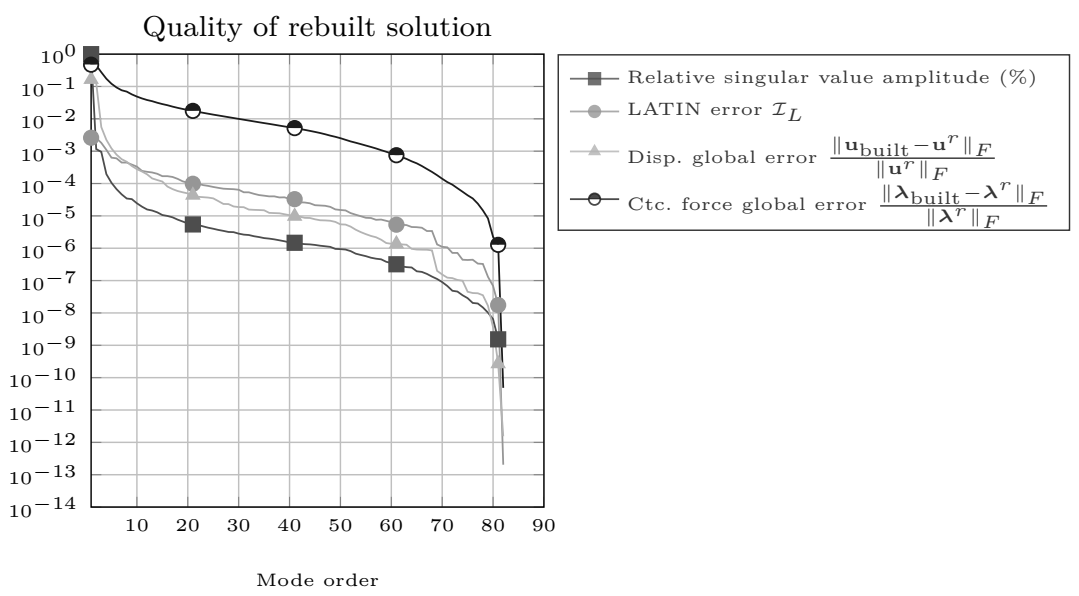

Figure 4: Errors of rebuilt solution taking into account a given number of modes.

$$
\mathbf{W}_{1}^{\mathrm{r}} v_{1}^{\mathrm{r}}(t)+\cdots+\mathbf{W}_{K}^{\mathrm{r}} v_{K}^{\mathrm{r}}(t)=\operatorname{svd}\left(\left(\boldsymbol{\lambda}^{\mathrm{r}}-\boldsymbol{\lambda}_{0}\right)+\mathbf{k B}\left(\mathbf{u}^{\mathrm{r}}-\mathbf{u}_{0}\right)\right)
$$

Associated vectors $\left(\mathbf{V}_{k}^{\mathrm{r}}\right)_{1}^{P}$ and $\left(\mathbf{L}_{k}^{\mathrm{r}}\right)_{1}^{P}$ correcting the elastic solution can be also computed and an approximated solution $\left(\tilde{\mathbf{u}}^{\mathrm{r}}, \tilde{\boldsymbol{\lambda}}^{\mathrm{r}}\right)$ can be rebuilt taking into account only $P$ modes. The larger is $P$, the more accurate is the solution (if $P=K$ the solution is exactly recovered). On figure 4 different error indicators are provided to illustrate the reducibility of the solution. For instance, if one takes into account 10 modes, the error on the displacement field is $2.7 \times 10^{-4}$, on the contact force field $4.8 \times 10^{-2}$ and the LATIN error measure is $3.3 \times 10^{-4}$. All in all, depending on the field of interest, very few modes are sufficient to generate the solution. Usually, the displacement field needs less modes than the contact force field to be recovered.

A few space modes for both displacement and contact force field and associated time modes are given on figure 5. The same observations as in [45] can be made as far as the scale separability of a frictional contact problem is concerned: First modes (the most energetic ones) depict a global scale of the solution (scale of the structure and spread over the whole time interval) whereas higher order modes bring corrections localized at the contacting interface and at specific time steps.

\subsection{Application of the quasi-optimal LATIN-PGD method}

In this section, performances are compared between the LATIN method (without separated representation) and the LATIN-PGD (see figure 6). To reach the same level of precision, the LATIN-PGD requires less iterations than the LATIN method. Moreover, note that the complexity of one LATIN-PGD iteration is far less than one of the LATIN method. So the computation cost is reduced drastically using the LATIN-PGD.

A very interesting point is the evolution of the PGD basis. As the LATINPGD converges to the solution of the reference problem, the PGD basis has to span the same subspace as the one given by (42). Remarkably, the size of the 

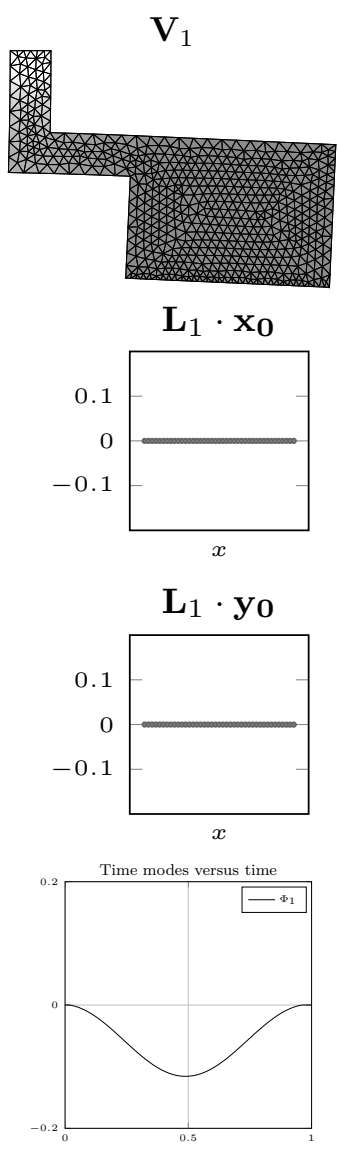

(a)
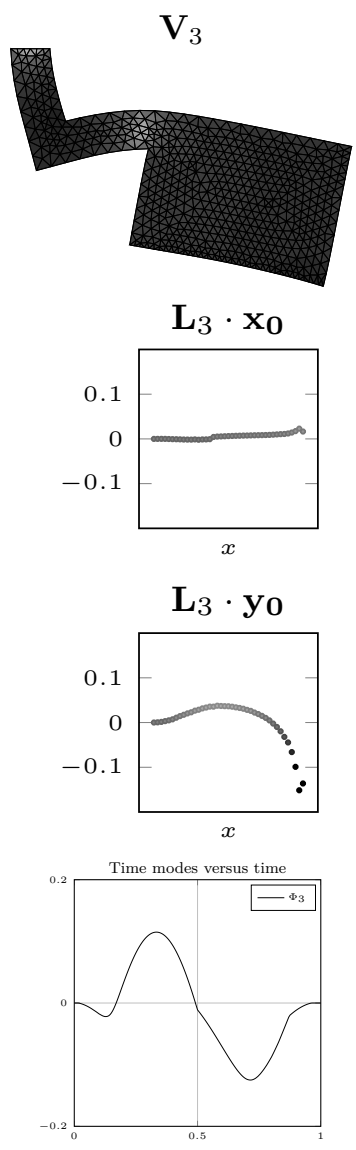

(b)
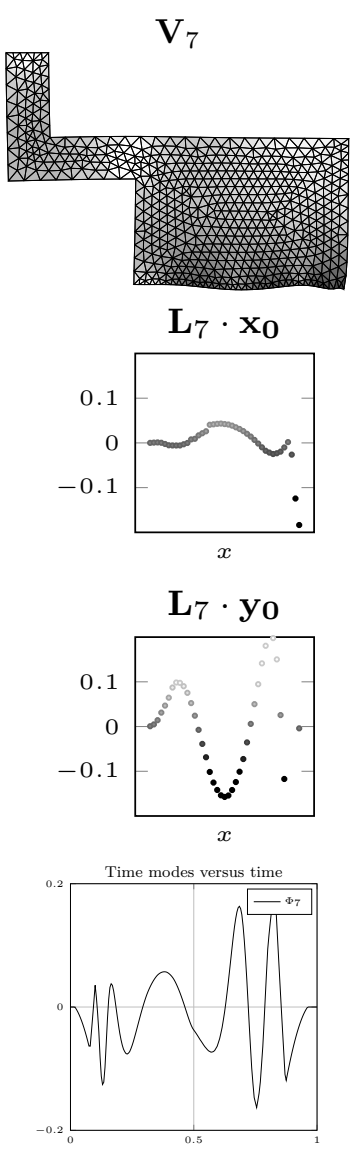

(c)

Figure 5: Space modes $\mathbf{V}_{1,3,7}$ (displacement field), $\mathbf{L}_{1,3,7}$ (contact force field) computed from $\mathbf{W}_{1,3,7}$ and time modes $\phi_{1,3,7}(t)$.

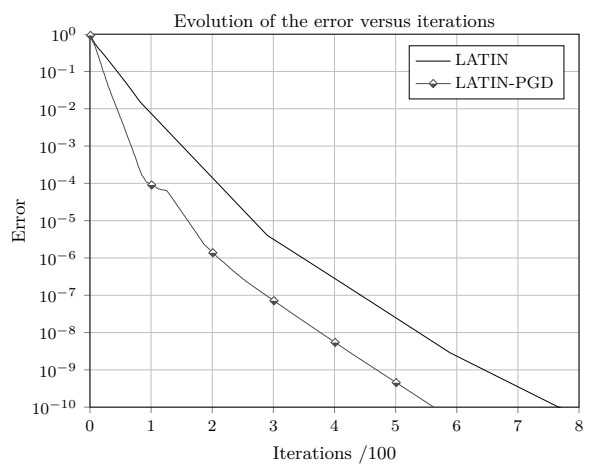

(a)

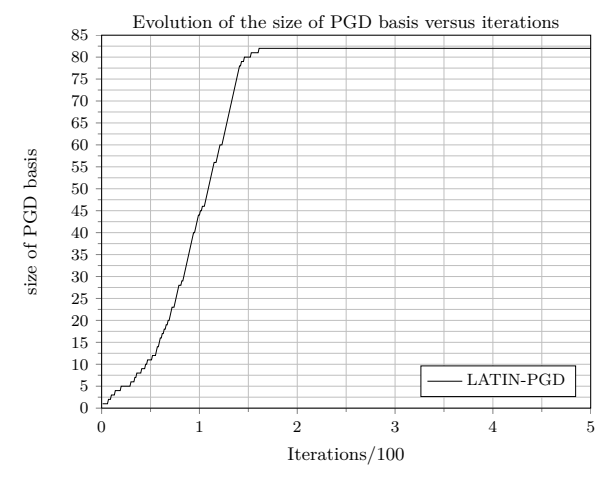

(b)

Figure 6: (a) Error given by the formula (26) versus iterations - (b) Size of the PGD basis versus iterations. 
PGD basis grows up until reaching, after a certain number of iterations, the size of the reference SVD basis.

The behavior of the PGD basis along iterations can be illustrated using Modal Assurance Criterion (MAC) diagrams [5]. Given two sets of vectors of same dimension $\left(\mathbf{X}_{i}\right)_{1}^{p}$ and $\left(\mathbf{Y}_{i}\right)_{1}^{q}$, the MAC matrix $\mathbf{M}$ is defined as follows:

$$
(\mathbf{M})_{\substack{1 \leqslant i \leqslant p \\ 1 \leqslant j \leqslant q}}=\frac{\left|\mathbf{X}_{i}^{T} \mathbf{Y}_{j}\right|^{2}}{\left\|\mathbf{X}_{i}\right\|^{2}\left\|\mathbf{Y}_{j}\right\|^{2}} \in[0,1]
$$

The coefficient $M_{i j}$ measures the correlation between modes $\mathbf{X}_{i}$ and modes $\mathbf{Y}_{j}$. If $M_{i j}=1$, then $\mathbf{X}_{i}$ and $\mathbf{Y}_{j}$ are colinear (highly correlated). On the contrary, $M_{i j}=0$ means that $\mathbf{X}_{i}$ and $\mathbf{Y}_{j}$ are orthogonal (uncorrelated). In practice MAC matrix is plotted using gray scale or $3 \mathrm{D}$ histogram to visualize correlation between a given set of modes and a reference one. Figure 7 reports the MAC matrices between the first 30 reference SVD modes and iterated PGD ones. The main observations can be pointed out:

- The PGD modes are clearly correlated to the corresponding SVD modes (according their order and depending on the precision of the solution).

- The PGD modes are computed hierarchically: structural modes are found at first.

- The larger the number of iterations is, the more accurate the solution is and the more correlations between PGD modes and SVD modes appear.

Thanks to the downsizing stage, iterated solutions are provided in a quasioptimal separated form (i.e. close to the SVD). To make it even more close to the reference SVD basis, the parameter $\xi_{\max }$ can be set to a higher value. But and as it was explained in section 3.3 , the strict optimality property is superfluous.

This observation emphasizes and exemplify a multiscale approach within the LATIN-PGD. The problem is firstly solved on the structural scales. Then, the problem is solved locally at the contacting interface. This strengthens the approach developed in [45] and fosters multiscale methods.

\subsection{Initialization using coarse computations}

In this section, a pre-computation method is proposed to initialize the PGD basis to accelerate convergence of the LATIN-PGD. It consists in finding global scale PGD modes (both in space and time) using a coarse space-time discretization (see figure 8). Indeed, the global scale behavior of the structure can be tracked using a coarse mesh and larger time steps. This pre-computation (less expensive than the reference model) allows to give a first guess of PGD modes. For the present example, we propose to use the coarse model described in the figure 9 and in the table 3 .

Once the coarse problem is solved (i.e. the solution $\mathbf{s}^{C}=\left(\mathbf{u}^{C}, \boldsymbol{\lambda}^{C}\right)$ is known), the displacement field is interpolated (using [39] or a trilinear interpolation ...) on the reference space-time discretization providing $\tilde{\mathbf{u}}$. Then, a first guess of the PGD basis can be deduced easily using the following formula:

$$
\widetilde{\mathbf{W}} \widetilde{\boldsymbol{\Phi}}^{T}=\operatorname{svd}\left(\mathbf{B K}\left(\tilde{\mathbf{u}}-\mathbf{u}_{0}\right)+\mathbf{k B}\left(\tilde{\mathbf{u}}-\mathbf{u}_{0}\right)\right)
$$



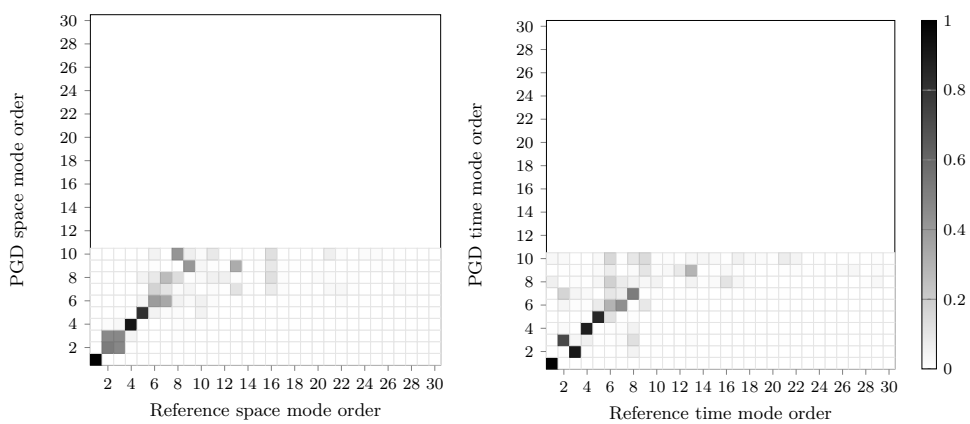

(a) PGD basis sizing 10
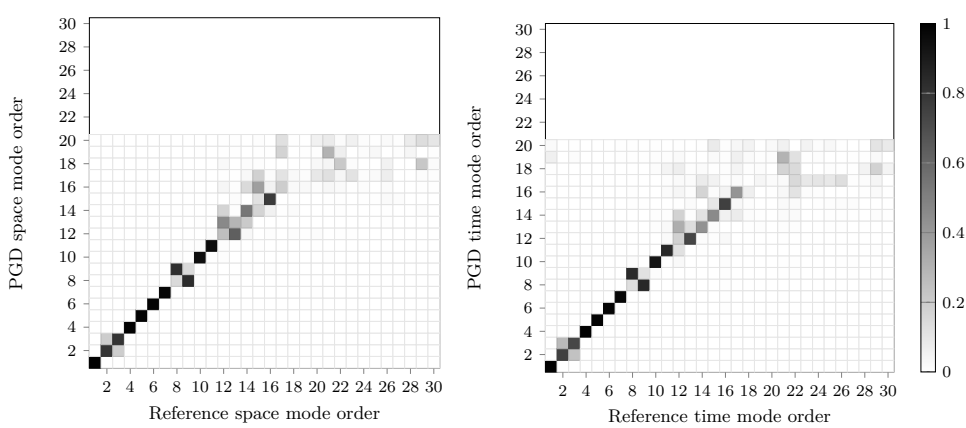

(b) PGD basis sizing 20
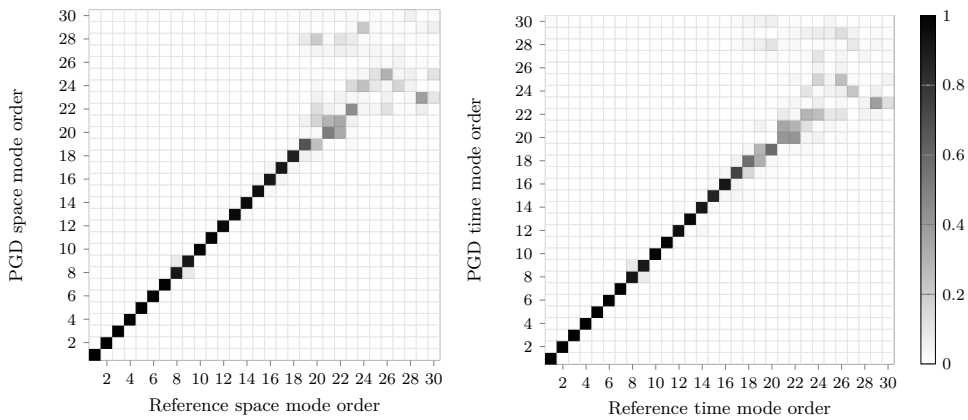

(c) PGD basis sizing 30

Figure 7: MAC diagrams after a certain number of iterations for both space modes and time modes.

\begin{tabular}{|c|c|c|}
\hline Number of time steps & $m$ & 50 \\
\hline Number of dofs & $n$ & 586 \\
\hline Number of contacting nodes & $n_{C}$ & 26 \\
\hline Final time & $T$ & $1 \mathrm{~s}$ \\
\hline Number of reduced SVD modes & & 40 \\
\hline
\end{tabular}

Table 3: Simulation parameters for the coarse model. 


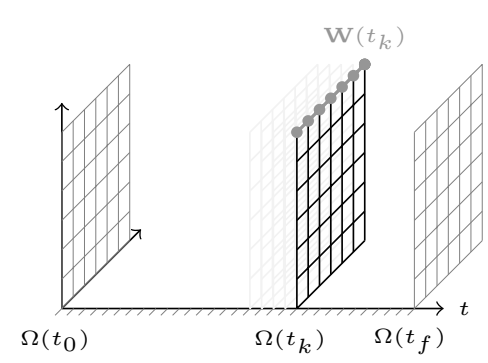

(a) Reference space-time discretizations

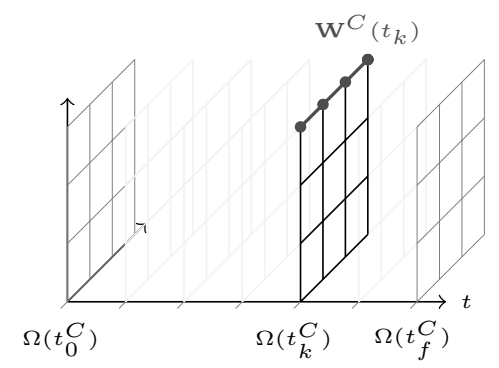

(b) Coarse space-time discretizations

Figure 8: A cheap pre-computation on coarse discretizations can be used to catch global time-pace modes to initialize LATIN-PGD on the fine reference discretizations.

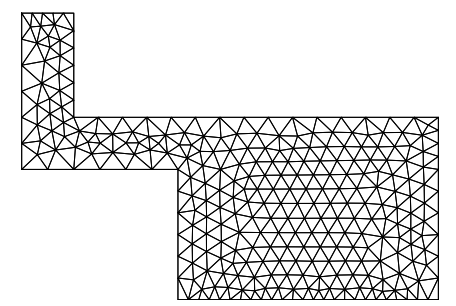

Figure 9: Coarse mesh used for pre-computation.

Only first modes of (44) are considered (global modes) because higher order ones may be irrelevant (dependence on the discretization and the interpolation method). This initialization (see figure 10) provides interesting features. First, the initial solution is more accurate than the elastic solution (roughly 3 decades are gained). Second, the error of the initialized LATIN-PGD decreases rapidly during first iterations until recovering the same convergence rate as the uninitialized LATIN-PGD (pre-computed coarse modes are rapidly corrected on the fine discretization). In practice, to reach a very high-level of accuracy (says $10^{-4}$ ), this initialization allows to divide by 2 the number of iterations (global modes are already found and few iterations are needed to capture the local behavior) relatively to the standard LATIN-PGD and by 4 relatively to the LATIN method. Nevertheless for higher levels of accuracy, more computational work is needed to describe precisely the local behavior diminishing relatively the gain of this initialization method. Of course, the gain of the initialization depends on the quality of the displacement projector from coarse to fine mesh.

\section{Three-dimensional numerical application}

The previous example can be easily transposed to a three dimensional case as described in the figure 11 and in the table 4 .

Same a posteriori analyses can be carried out for the solution to exhibit the multiscale content of the SVD basis (see figures 12 and 13). As the previous 


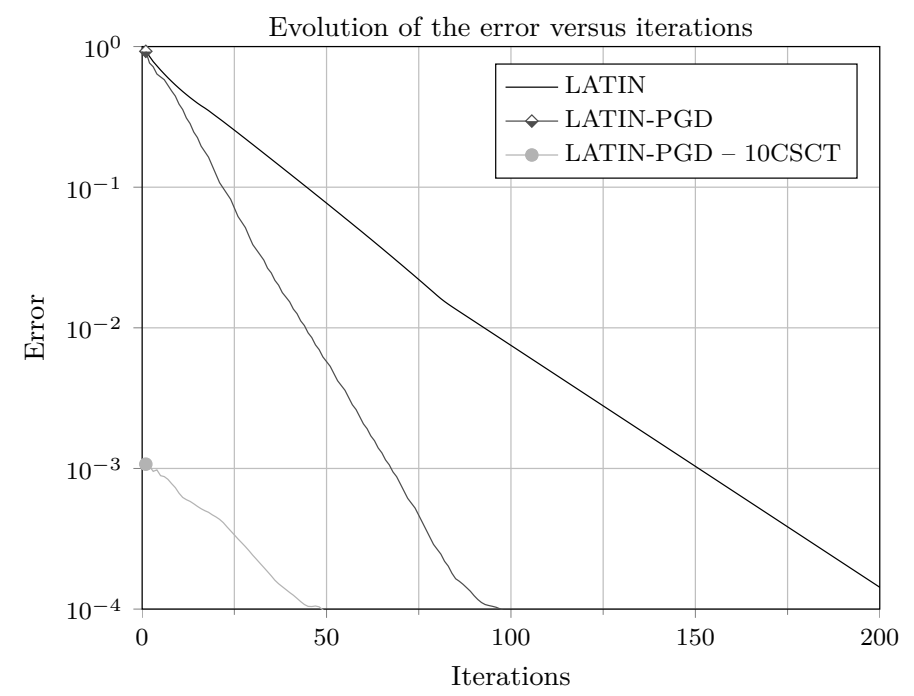

Figure 10: Convergence plots with an initial guess for the PGD basis.

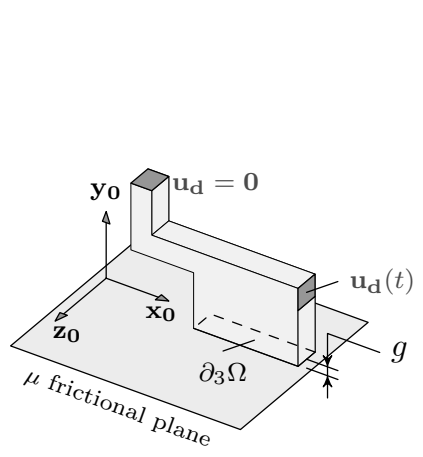

(a)

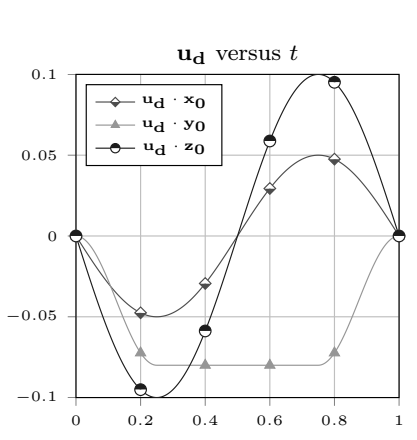

(b)

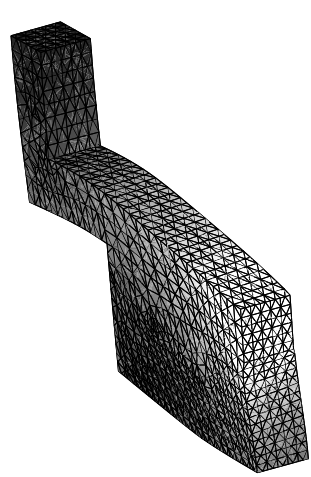

(c)

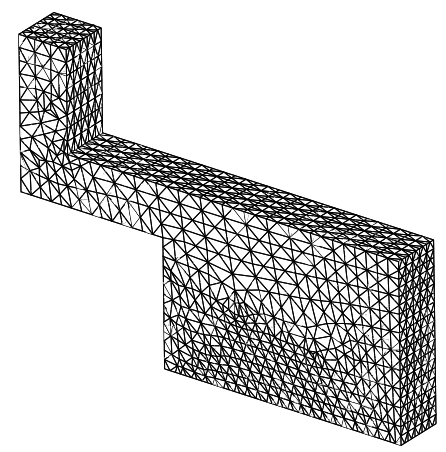

(d)

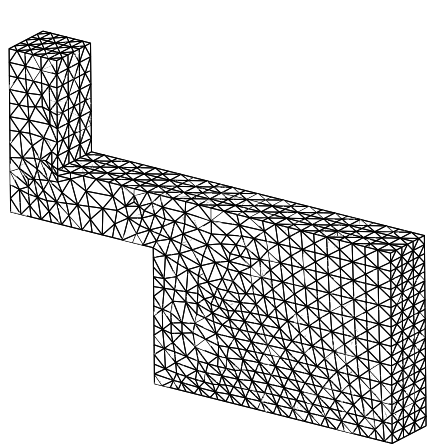

(e)

Figure 11: (a) Model - (b) Prescribed displacement evolution - (c) Shape of the deformed body (fine mesh) at $t_{150}-$ (d) Fine mesh - (e) Coarse mesh. 


\begin{tabular}{|c|c|c|c|}
\hline & & Fine model & Coarse model \\
\hline Number of time steps & $m$ & 200 & 50 \\
\hline Number of dofs & $n$ & 12099 & 7920 \\
\hline Number of contacting nodes & $n_{C}$ & 385 & 172 \\
\hline Young modulus & $E$ & $10^{6} \mathrm{~Pa}$ & $10^{6} \mathrm{~Pa}$ \\
\hline Poisson ratio & $\nu$ & 0.3 & 0.3 \\
\hline Friction coefficient & $\mu$ & 0.15 & 0.15 \\
\hline Final time & $T$ & $1 \mathrm{~s}$ & $1 \mathrm{~s}$ \\
\hline Number of reduced SVD modes & & 160 & 44 \\
\hline Search direction & $k_{T}$ & $3000 \mathrm{~N} / \mathrm{m}$ & $3000 \mathrm{~N} / \mathrm{m}$ \\
\hline Search direction & $k_{N}$ & $1000 \mathrm{~N} / \mathrm{m}$ & $1000 \mathrm{~N} / \mathrm{m}$ \\
\hline
\end{tabular}

Table 4: Simulation parameters.

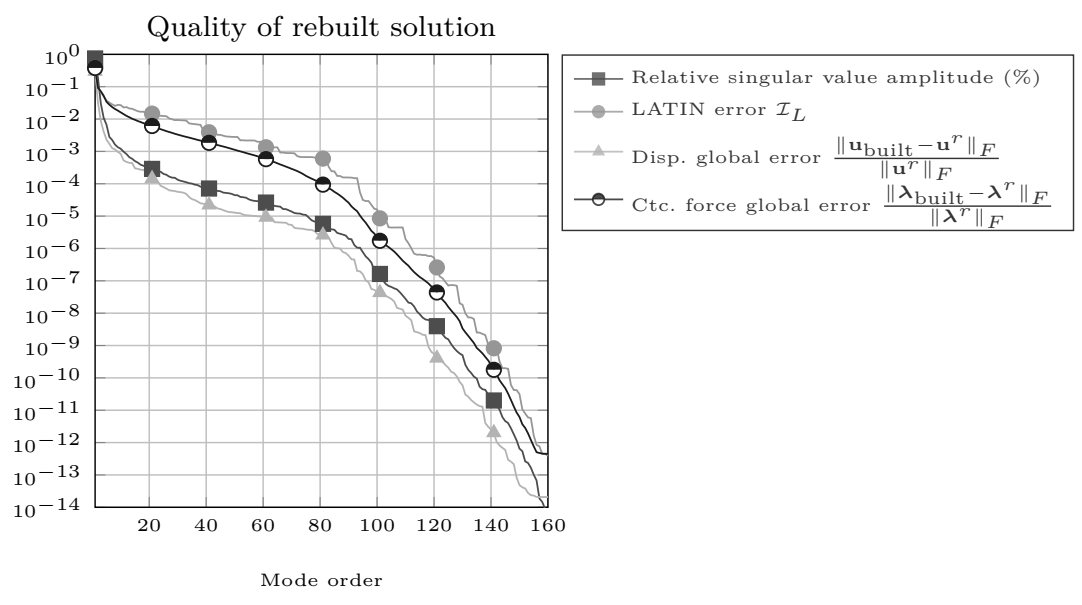

Figure 12: Errors of rebuilt solution taking into account a given number of modes for the 3D problem.

two-dimensional problem, a scale separability phenomenon can be pointed out as far as spatial and time modes are concerned.

The LATIN method, the LATIN-PGD and the LATIN-PGD initialized with 10 coarse space-time modes are compared on figure 14. LATIN-PGD overtakes again the LATIN method in terms of iterations. In addition, a LATIN-PGD iteration is less costly than one of the LATIN method. The initialization of the PGD basis allows to gain roughly one decade which is limited in comparison to the two dimensional previous example. To achieve it and for sake of simplicity, the authors use a collocation projection which is simpler but less accurate than [39]. Nevertheless, the LATIN-PGD shows a great potential to solve 3D frictional problems well-known for their toughness. 

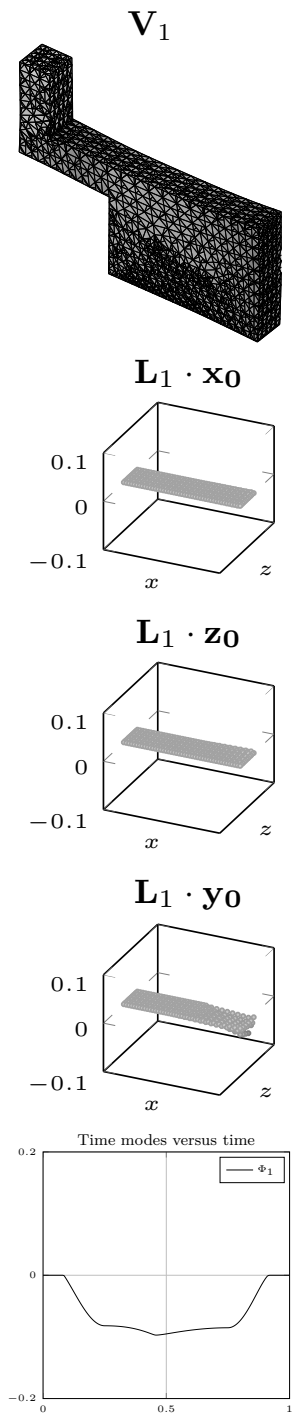

(a)
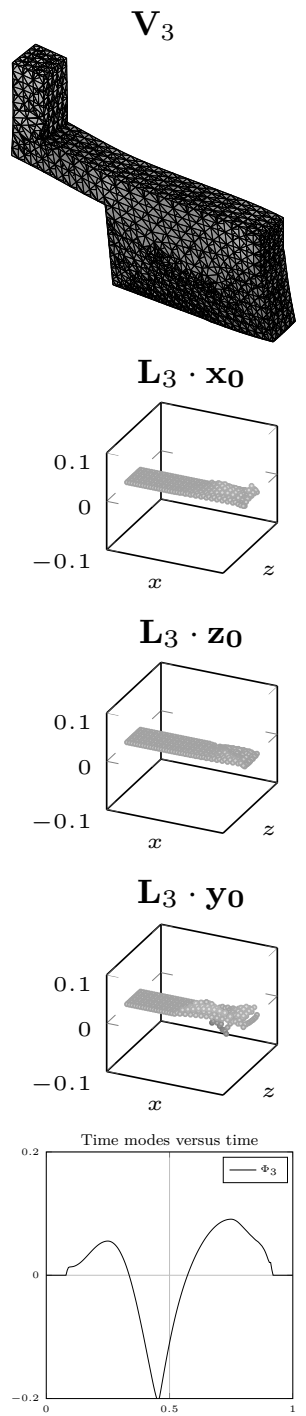

(b)
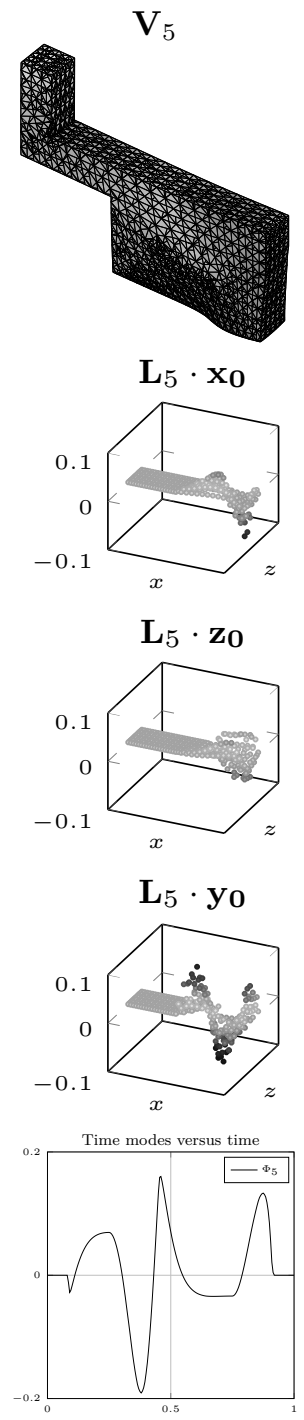

(c)

Figure 13: Space-time modes $\mathbf{V}_{1,3,5}$ (displacement field), $\mathbf{L}_{1,3,5}$ (contact force field) computed from $\mathbf{W}_{1,3,5}$ and $\phi_{1,3,5}(t)$ for the $3 \mathrm{D}$ problem. 


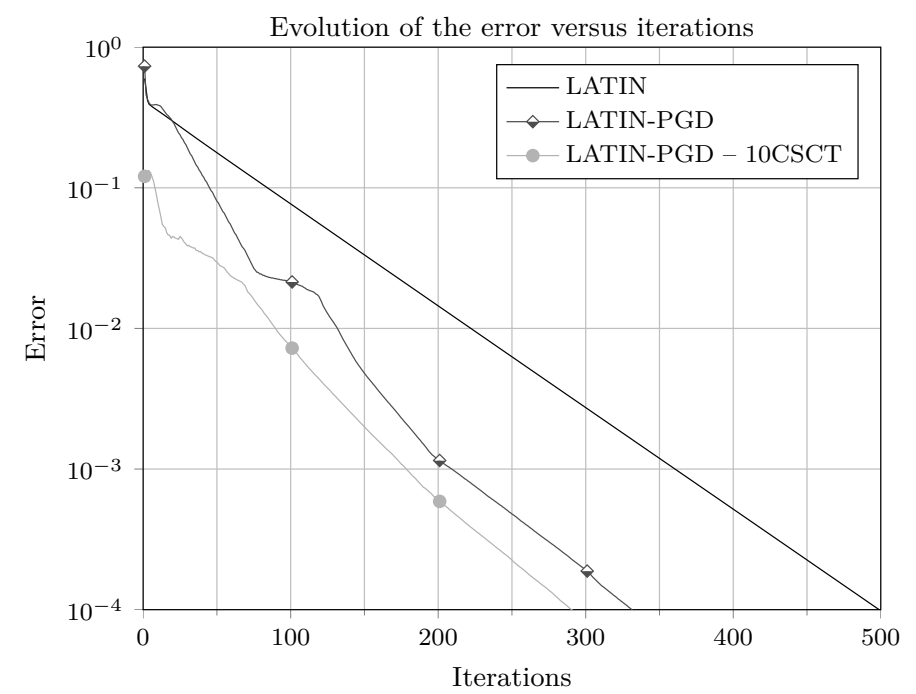

Figure 14: Convergence plots with an initial guess for the PGD basis for the 3D problem.

\section{Conclusion and perspectives}

This article provides the formulation of the quasi-optimal LATIN-PGD solver dedicated to frictional contact problems. This new a priori reduced basis strategy converges faster compared to classic LATIN solver and requires less computational effort and memory usage. Moreover, the quasi-optimality property is a striking observation. The solution is found progressively according to its optimal modes provided by the SVD. This is achieved by a enhanced preliminary stage increasing the convergence rate and an efficient downsizing stage awarding the suggested approach a quasi-optimality property for PGD basis. At convergence, the solution of the considered problem is provided into a separated space-time form close to the SVD.

Numerical applications illustrate the ability of the suggested approach to solve two-dimensional and three-dimensional problems. In addition and to take advantage of the scale separability of the space-time basis, an initialization method was proposed. Global and structural modes of the solution are computed on coarse space-time discretizations. Then, they are reused to provide an initial guess for the space-time basis on reference discretizations sparing a consequent computational effort.

In this paper, the tackled problem involves a single flexible body contacting a rigid basement for the sake of simplicity. Nonetheless, multibody system can be easily implemented by considering appropriate frictional contact conditions between a couple of flexible bodies. So, the local stage has to be generalized to these new conditions. But, the overall of the approach remains the same. The global stage aims still at solving internal balance with PGD space-time representation but different approaches are possible. The first consists in tackling globally all solids with a single family of space-time functions wherein whole unknowns are assembled. The second consists in tackling each solid individually 
with a dedicated family of space-time functions. This last approach is suited for parallel computing but harder to implement.

Furthermore, the LATIN method provides also a framework to take into account other kind of non-linearities such as finite strains, non-linear material such as plasticity accounting the space-time separation. Then, it would be possible to take into account those supplementary non-linearities in the presented work with the developed method.

This strategy can be easily extended to the multiparametric framework as introduced in $[26,49]$. For that purpose, a parameter of the reference problem (e.g. frictional coefficient) may considered as a variable of the problem. Then, the approximated displacement field solution is still sought as a separated form solution $\mathbf{u}(\mathbf{x}, t ; \mu)=\mathbf{u}(\mathbf{x}, t, \mu)=\mathbf{u}_{0}(\mathbf{x}, t, \mu)+\sum_{i} \mathbf{V}(\mathbf{x}) \phi(t) f(\mu)$. Nevertheless, downsizing approaches and compression techniques such as PARAFAC [19, 20] or promising HOSVD $[64,55,33,65]$ remain challenging.

\section{Acknowledgment}

We gratefully acknowledge the French National Association for Research and Technology (ANRT, CIFRE grant number 2011/0705).

\section{References}

[1] P. Alart. Méthode de newton généralisée en mécanique du contact. Journal de mathématiques pures et appliquées, 76(1):83-108, 1997.

[2] P. Alart and D. Dureisseix. A scalable multiscale latin method adapted to nonsmooth discrete media. Computer Methods in Applied Mechanics and Engineering, 197(5):319-331, 2008.

[3] P. Alart, D. Dureisseix, and M. Renouf. Using Nonsmooth Analysis for Numerical Simulation of Contact Mechanics, volume 12 of Advances in Mechanics and Mathematics. Springer US, 2006.

[4] P. Alart and F. Lebon. Solution of frictional contact problems using ilu and coarse/fine preconditioners. Computational mechanics, 16(2):98-105, 1995.

[5] R. J. Allemang. The modal assurance criterion-twenty years of use and abuse. Sound and vibration, 37(8):14-23, 2003.

[6] D. Amsallem and C. Farhat. Interpolation method for adapting reducedorder models and application to aeroelasticity. AIAA journal, 46(7):18031813, 2008.

[7] D. Amsallem and C. Farhat. An online method for interpolating linear parametric reduced-order models. SIAM Journal on Scientific Computing, 33(5):2169-2198, 2011.

[8] D. Amsallem, M. J. Zahr, and C. Farhat. Nonlinear model order reduction based on local reduced-order bases. International Journal for Numerical Methods in Engineering, 92(10):891-916, 2012. 
[9] M. Anitescu and F. A. Potra. Formulating dynamic multi-rigid-body contact problems with friction as solvable linear complementarity problems. Nonlinear Dynamics, 14(3):231-247, 1997.

[10] C. G. Baker, K. A. Gallivan, and P. Van Dooren. Low-rank incremental methods for computing dominant singular subspaces. Linear Algebra and its Applications, 436(8):2866-2888, 2012.

[11] M. Barboteu, P. Alart, and M. Vidrascu. A domain decomposition strategy for nonclassical frictional multi-contact problems. Computer Methods in Applied Mechanics and Engineering, 190(37):4785-4803, 2001.

[12] G. Berkooz, P. Holmes, and J. L. Lumley. The proper orthogonal decomposition in the analysis of turbulent flows. Annual review of fluid mechanics, 25(1):539-575, 1993.

[13] P. Boisse, P. Bussy, and P. Ladevèze. A new approach in non-linear mechanics: The large time increment method. International journal for numerical methods in engineering, 29(3):647-663, 1990.

[14] P. A. Boucard and L. Champaney. A suitable computational strategy for the parametric analysis of problems with multiple contact. International Journal for Numerical Methods in Engineering, 57(9):1259-1281, 2003.

[15] P.-A. Boucard, P. Ladevèze, M. Poss, and P. Rougée. A nonincremental approach for large displacement problems. Computers 83 structures, 64(1):499-508, 1997.

[16] L. Boucinha, A. Gravouil, and A. Ammar. Space-time proper generalized decompositions for the resolution of transient elastodynamic models. Computer Methods in Applied Mechanics and Engineering, 255:67 - 88, 2013.

[17] M. Brand. Incremental singular value decomposition of uncertain data with missing values. In Computer VisionECCV 2002, pages 707-720. Springer, 2002.

[18] M. Brand. Fast low-rank modifications of the thin singular value decomposition. Linear algebra and its applications, 415(1):20-30, 2006.

[19] R. Bro. Parafac. tutorial and applications. Chemometrics and intelligent laboratory systems, 38(2):149-171, 1997.

[20] R. Bro and H. A. Kiers. A new efficient method for determining the number of components in parafac models. Journal of chemometrics, 17(5):274-286, 2003.

[21] J. R. Bunch and C. P. Nielsen. Updating the singular value decomposition. Numerische Mathematik, 31(2):111-129, 1978.

[22] P. Bussetta, D. Marceau, and J.-P. Ponthot. The adapted augmented lagrangian method: a new method for the resolution of the mechanical frictional contact problem. Computational Mechanics, 49(2):259-275, 2012. 
[23] K. Carlberg, C. Farhat, J. Cortial, and D. Amsallem. The gnat method for nonlinear model reduction: effective implementation and application to computational fluid dynamics and turbulent flows. Journal of Computational Physics, 242:623-647, 2013.

[24] L. Champaney, J.-Y. Cognard, D. Dureisseix, and P. Ladevèze. Large scale applications on parallel computers of a mixed domain decomposition method. Computational Mechanics, 19:253-263, 1997.

[25] L. Champaney, J.-Y. Cognard, and P. Ladevèze. Modular analysis of assemblages of three-dimensional structures with unilateral contact conditions. Computers and Structures, 73:249-266, 1999.

[26] F. Chinesta, A. Ammar, and E. Cueto. Recent advances and new challenges in the use of the proper generalized decomposition for solving multidimensional models. Archives of Computational methods in Engineering, 17(4):327-350, 2010.

[27] F. Chinesta, A. Ammar, A. Leygue, and R. Keunings. An overview of the proper generalized decomposition with applications in computational rheology. Journal of Non-Newtonian Fluid Mechanics, 166(11):578-592, 2011.

[28] F. Chinesta, R. Keunings, and A. Leygue. The Proper Generalized Decomposition for Advanced Numerical Simulations. Springer International Publishing, 2014.

[29] F. Chinesta, P. Ladevèze, and E. Cueto. A short review on model order reduction based on proper generalized decomposition. Archives of Computational Methods in Engineering, 18:395-404, 2011.

[30] J.-Y. Cognard and P. Ladevèze. A large time increment approach for cyclic viscoplasticity. International journal of plasticity, 9(2):141-157, 1993.

[31] C.-A. Coulomb. Théorie des machines simples. Mémoires de l'Académie des Sciences, 1785.

[32] A. Curnier and P. Alart. A generalized newton method for contact problems with friction. Journal de mécanique théorique et appliquée, 7:67-82, 1988.

[33] L. De Lathauwer, B. De Moor, and J. Vandewalle. A multilinear singular value decomposition. SIAM journal on Matrix Analysis and Applications, 21(4):1253-1278, 2000.

[34] G. De Saxcé and Z.-Q. Feng. The bipotential method: a constructive approach to design the complete contact law with friction and improved numerical algorithms. Mathematical and Computer Modelling, 28(4):225$245,1998$.

[35] J. Dolbow, N. Moës, and T. Belytschko. An extended finite element method for modeling crack growth with frictional contact. Computer Methods in Applied Mechanics and Engineering, 190(51):6825-6846, 2001. 
[36] Z. Dostál, T. Kozubek, P. Horyl, T. Brzobohatỳ, and A. Markopoulos. A scalable tfeti algorithm for two-dimensional multibody contact problems with friction. Journal of computational and applied mathematics, 235(2):403-418, 2010.

[37] Z. Dostál, T. Kozubek, A. Markopoulos, T. Brzobohatỳ, V. Vondrák, and P. Horyl. A theoretically supported scalable tfeti algorithm for the solution of multibody 3d contact problems with friction. Computer Methods in Applied Mechanics and Engineering, 205:110-120, 2012.

[38] Z. Dostál and J. Schoberl. Minimizing quadratic functions subject to bound constraints with the rate of convergence and finite termination. Computational Optimization and Applications, 30(1):23-43, 2005.

[39] D. Dureisseix and H. Bavestrello. Information transfer between incompatible finite element meshes: application to coupled thermo-viscoelasticity. Computer Methods in Applied Mechanics and Engineering, 195(44):65236541, 2006.

[40] D. Dureisseix and C. Farhat. A numerically scalable domain decomposition method for the solution of frictionless contact problems. International Journal for Numerical Methods in Engineering, 50(12):2643-2666, 2001.

[41] C. Eckart and G. Young. The approximation of one matrix by another of lower rank. Psychometrika, 1:211-218, 1936.

[42] M. Fortin and R. Glowinski. Augmented Lagrangian methods: applications to the numerical solution of boundary-value problems. Access Online via Elsevier, 2000.

[43] F. Galland. An adaptive model reduction approach for 3D fatigue crack growth in small scale yielding conditions. PhD thesis, INSA de Lyon, 2011.

[44] F. Galland, A. Gravouil, E. Malvesin, and M. Rochette. A global model reduction approach for $3 \mathrm{~d}$ fatigue crack growth with confined plasticity. Computer Methods in Applied Mechanics and Engineering, 200(5):699-716, 2011.

[45] A. Giacoma, D. Dureisseix, A. Gravouil, and M. Rochette. A multiscale large time increment/fas algorithm with time-space model reduction for frictional contact problems. International Journal for Numerical Methods in Engineering, 97(3):207-230, 2014.

[46] G. H. Golub and C. Reinsch. Singular value decomposition and least squares solutions. Numerische Mathematik, 14(5):403-420, 1970.

[47] G. H. Golub and C. F. Van Loan. Matrix computations, volume 3. JHU Press, 2012.

[48] M. Heinstein and T. A. Laursen. An algorithm for the matrix-free solution of quasistatic frictional contact problems. International Journal for Numerical Methods in Engineering, 44(9):1205-1226, 1999. 
[49] C. Heyberger, P.-A. Boucard, and D. Néron. Multiparametric analysis within the proper generalized decomposition framework. Computational Mechanics, 49(3):277-289, 2012.

[50] C. Heyberger, P.-A. Boucard, and D. Néron. A rational strategy for the resolution of parametrized problems in the PGD framework. Computer Methods in Applied Mechanics and Engineering, 259:40-49, 2013.

[51] M. Jean. The non-smooth contact dynamics method. Computer methods in applied mechanics and engineering, 177(3):235-257, 1999.

[52] P. Joli and Z.-Q. Feng. Uzawa and newton algorithms to solve frictional contact problems within the bi-potential framework. International journal for numerical methods in engineering, 73(3):317-330, 2008.

[53] F. Jourdan, P. Alart, and M. Jean. A gauss-seidel like algorithm to solve frictional contact problems. Computer methods in applied mechanics and engineering, 155(1):31-47, 1998.

[54] P. Kerfriden, P. Gosselet, S. Adhikari, and S. Bordas. Bridging proper orthogonal decomposition methods and augmented newton-krylov algorithms: An adaptive model order reduction for highly nonlinear mechanical problems. Computer Methods in Applied Mechanics and Engineering, 200:850 - 866, 2011.

[55] T. G. Kolda and B. W. Bader. Tensor decompositions and applications. SIAM review, 51(3):455-500, 2009.

[56] P. Ladevèze. Nonlinear Computational Structural Methods: New Approaches and Non-Incremental Methods of Calculation. Springer-Verlag New York Inc., 1999.

[57] P. Ladevèze and L. Chamoin. On the verification of model reduction methods based on the proper generalized decomposition. Computer Methods in Applied Mechanics and Engineering, 200(23):2032-2047, 2011.

[58] P. Ladevèze, H. Lemoussu, and P. Boucard. A modular approach to 3-D impact computation with frictional contact. Computers and Structures, $78: 45-51,2000$.

[59] P. Ladevèze, J.-C. Passieux, and D. Néron. The LATIN multiscale computational method and the proper generalized decomposition. Computer Methods in Applied Mechanics and Engineering, 199:1287 - 1296, 2010.

[60] T. A. Laursen. Formulation and treatment of frictional contact problems using finite elements. PhD thesis, Stanford University, 1992.

[61] F. Lebon, M. Raous, and I. Rosu. Multigrid methods for unilateral contact problems with friction. In IUTAM Symposium on Computational Methods in Contact Mechanics, volume 3, pages 1-16. Springer, 2007.

[62] H. Lemoussu, P.-A. Boucard, and P. Ladevèze. A 3D shock computational strategy for real assembly and shock attenuator. Advances in Engineering Software, 33:517 - 526, 2002. 
[63] D. Li and M. Fukushima. Smoothing newton and quasi-newton methods for mixed complementarity problems. Computational Optimization and Applications, 17(2-3):203-230, 2000.

[64] D. Luo, C. Ding, and H. Huang. Are tensor decomposition solutions unique? on the global convergence hosvd and parafac algorithms. In Advances in Knowledge Discovery and Data Mining, pages 148-159. Springer, 2011.

[65] X. Ma, D. Schonfeld, and A. Khokhar. Dynamic updating and downdating matrix svd and tensor hosvd for adaptive indexing and retrieval of motion trajectories. In Acoustics, Speech and Signal Processing, 2009. ICASSP 2009. IEEE International Conference on, pages 1129-1132. IEEE, 2009.

[66] S. Niroomandi, I. Alfaro, E. Cueto, and F. Chinesta. Real-time deformable models of non-linear tissues by model reduction techniques. Computer Methods and Programs in Biomedicine, 91(3):223-231, 2008.

[67] A. Nouy. A priori model reduction through proper generalized decomposition for solving time-dependent partial differential equations. Computer Methods in Applied Mechanics and Engineering, 199(23):1603-1626, 2010.

[68] D. Odièvre, P.-A. Boucard, and F. Gatuingt. A parallel, multiscale domain decomposition method for the transient dynamic analysis of assemblies with friction. Computer Methods in Applied Mechanics and Engineering, 199(21):1297-1306, 2010.

[69] G. Pietrzak and A. Curnier. Large deformation frictional contact mechanics: continuum formulation and augmented lagrangian treatment. Computer Methods in Applied Mechanics and Engineering, 177(3):351-381, 1999.

[70] N. Relun, D. Néron, and P. Boucard. A model reduction technique based on the pgd for elastic-viscoplastic computational analysis. Computational Mechanics, 51(1):83-92, 2013.

[71] M. Renouf and P. Alart. Conjugate gradient type algorithms for frictional multi-contact problems: applications to granular materials. Computer Methods in Applied Mechanics and Engineering, 194(18):2019-2041, 2005.

[72] R. Ribeaucourt, M.-C. Baietto-Dubourg, and A. Gravouil. A new fatigue frictional contact crack propagation model with the coupled XFEM/LATIN method. Computer Methods in Applied Mechanics and Engineering, 196:3230 - 3247, 2007.

[73] D. Ryckelynck. A priori hyperreduction method: an adaptive approach. Journal of Computational Physics, 202(1):346-366, 2005.

[74] D. Ryckelynck, F. Chinesta, E. Cueto, and A. Ammar. On the a priori model reduction: Overview and recent developments. Archives of Computational Methods in Engineering, 13:91-128, 2006.

[75] A. Signorini. Questioni di elasticità non linearizzata e semilinearizzata. Rendiconti di Matematica e delle sue applicazioni, V. Ser., 18:95-139, 1959. 
[76] J. Simo and T. Laursen. An augmented lagrangian treatment of contact problems involving friction. Computers \&3 Structures, 42(1):97-116, 1992.

[77] B. Trollé, A. Gravouil, M.-C. Baietto, and T. Nguyen-Tajan. Optimization of a stabilized X-FEM formulation for frictional cracks. Finite Elements in Analysis and Design, 59:18 - 27, 2012.

[78] B. Wohlmuth. Variationally consistent discretization schemes and numerical algorithms for contact problems. Acta Numerica, 20:569-734, 2011.

[79] P. Wriggers. Finite element algorithms for contact problems. Archives of Computational Methods in Engineering, 2:1-49, 1995.

[80] V. A. Yastrebov. Numerical methods in contact mechanics. John Wiley \& Sons, 2013. 